\title{
Multivariate Pattern Analysis Utilizing Structural or Functional MRI - In Individuals With Musculoskeletal Pain and Healthy Controls: A Systematic Review
}

Ashley Smith ${ }^{1}$, Marina López-Solà ${ }^{2}$, Katie McMahon ${ }^{3}$, Ashley Pedler ${ }^{1}$, Michele Sterling ${ }^{1}$

\author{
${ }^{1}$ Recover Injury Research Centre, NHMRC CRE in Recovery Following Road Traffic Injury, Menzies \\ Health Institute QLD, Griffith University, Gold Coast, Australia \\ ${ }^{2}$ Cognitive and Affective Neuroscience Laboratory, Department of Psychology and Neuroscience \\ Institute of Cognitive Science, The University of Colorado, Boulder, USA \\ ${ }^{3}$ Centre for Advanced Imaging, University of Queensland, Herston, Australia
}

Keywords: musculoskeletal pain, functional MRI, multivariate pattern analysis, systematic review.

Correspondence concerning this article should be addressed to:

Ashley Smith, Griffith University, Gold Coast Campus, Southport QLD 4125.

E-mail: ashley.smith@griffith.edu.au, T: +61 755529564. 


\section{Introduction}

Musculoskeletal pain is highly prevalent and places a huge burden on society (Vos et al., 2012). It is postulated that treatment directed at the underlying mechanisms of pain would assist with more effective therapeutic outcomes (Baron, Binder, \& Wasner, 2012; Woolf, 2011). However, it is challenging to provide appropriately directed specific treatment when the underlying neurophysiological processes are not well understood. Recent studies report that chronic musculoskeletal pain conditions involve morphological (structural) brain changes (Apkarian, Hashmi, \& Baliki, 2011; Schmidt-Wilcke et al., 2006; Smallwood et al., 2013) and functional adaptations of brain processing (Baliki et al., 2012; Flor, Braun, Elbert, \& Birbaumer, 1997; Giesecke et al., 2004). As such, various non-invasive structural and functional neuroimaging techniques (e.g. Voxel-based morphometry, diffusion tensor imaging, functional magnetic resonance imaging (fMRI) and restingstate $\mathrm{fMRI}$ ) have been utilized to further understand cortical and sub-cortical processes associated with pain (Apkarian, 2011).

These methods allow recording of a large number of brain signals within a short period of time; either at rest, or following application of various stimuli. Historically, the results from these studies have been analysed using univariate techniques within the general linear model. Univariate analyses examine voxels independently to detect regional-average changes in activation, allowing determination of brain regions that are associated with various aspects of pain processing. As univariate analyses provide statistical analyses based on group differences between brain function/structure and behavioural outcomes (e.g. pain duration/frequency), these analyses are generally not as well suited at providing information at the individual level (Schmidt-Wilcke et al., 2006). Given that pain is an individual, multidimensional experience involving a distributed network of sensory, cognitive and evaluative processes (Clark, Yang, Tsui, Ng, \& Bennett Clark, 2002), the dynamic spatiotemporal relationship of pain provides challenges for neuroimaging. Recently, brain decoding, or multivariate pattern analyses (MVPA) has emerged to assist with analysis of the large sets of data through examination of combined signals of brain activity/structure across voxels (Haufe et al., 2014; Haxby et al., 2001). Underlying MVPA, are machine-learning techniques which allow determination of brain regions that may be contributing to prediction of pain reports from $\mathrm{FMRI}$ activity (Haynes \& Rees, 2006); or alternatively, determination of which voxel-based spatial and/or temporal patterns provide discrimination between two or more conditions (Mur, Bandettini, \& Kriegeskorte, 2009). This allows the potential of examining patterns of aberrant brain structure and/or function at an individual level, by examining distributed spatial patterns of brain activity/structure across conditions, rather than specific locations that are considered separately as measured by univariate analyses. Thus, MVPA could potentially assist in disentanglement of the respective contribution of various underlying brain processes associated with the pathogenesis and maintenance of musculoskeletal pain, and may provide a basis to allow specific treatment to be applied (Mackey, 2013; Tracey, 2011).

Although MVPA changes how pain is analysed and interpreted (Davis et al., 2014), and may provide a broader understanding of pain (Rogachov, Cheng, \& DeSouza, 2015), the overall utility and limitations of this analytic technique is unknown, and as such, a synthesis of the literature is warranted. The aim of this study was to systematically review the evidence relating to findings generated by MVPA following structural or fMRI to determine if this analysis is able to:

a) Discriminate between individuals with musculoskeletal pain and healthy controls,

b) Predict pain perception in healthy individuals stimulated with a noxious stimulus compared to those stimulated with a non-noxious stimulus. 


\section{Material and methods}

\subsection{Prospective registration of systematic review}

The protocol details of the systematic review were registered prospectively with PROSPERO international prospective register of systematic reviews (Registration Number: CRD42015025054) on August 6, 2015 and available from: http://www.crd.york.ac.uk/PROSPERO/display record.asp?ID=CRD42015025054

\subsection{Search methods for identification of studies}

Searches were conducted in the electronic databases MEDLINE (http://www.ncbi.nlm.nih.gov/entrez), CINAHL (1966-present), Embase (all years), PEDro, Google Scholar, Cochrane library (Cochrane reviews, Cochrane central register of controlled trials) and Web of Science (http://isiwebofknowledge.com). Reference lists and bibliographies of identified full text publications and review articles in the field were manually checked for additional studies. Full text studies published in English prior to $14^{\text {th }}$ of October 2016 were searched.

Key words related to pain, neuroimaging, multivariate pattern analysis and the brain were used as follows (Table 1). If available, corresponding medical subheadings (MeSH terms) were added for each search term in Medline, CINAHL, Embase, Cochrane and Web of Science. Search terms within each column were entered using the Boolean operator 'OR'. Following the completion of these searches, the search used the Boolean operator 'AND' to combine columns. Search strategies were customised for each database. For example, PEDro searches used the advanced search option to enter individual text words into the 'Abstract \& Title' Field, in addition to clinical trial being entered in the 'Method' field. For Google Scholar, no * were applied to the search terms.

\begin{tabular}{|l|l|l|l|}
\hline Population & Intervention & Comparison & Outcome \\
\hline Pain, Acute Pain, & Imag* & Multivaria* & Brain/pathology, \\
Chronic Pain & Scan* & Brain Decod*, Decod* & Cortical \\
Acute* & Neuro-imag*, & Machine Learn* & Cerebral Cortex \\
Chronic* & Neuroimag* & Artificial Intelligence & Neuroscience \\
Back & MRI, Magnetic & Computer-Assist* & Pain neuromatrix \\
Low Back* & resonance imag*, & & Neuroanatom* \\
Musculoskel* & fMRI, functional & & Neuroplastic* \\
Intractable* & magnetic resonance & & Neurological pain \\
Neck & imag* & signature, NPS. \\
Myofascial* & BOLD, blood oxygen- & & \\
Spine, Spin* & level dependent & & \\
Back-ache, Back*ache & contrast & & \\
Lumbago & Voxel-based & & \\
Fibromyalg* & morphometry, VBM & & \\
Whip-lash*, Whip*lash & Brain Map* & \\
Whiplash Injuries & & & \\
\hline
\end{tabular}

Table 1: Key Words

\subsection{Selection of studies}

In the first phase, the initial screening of retrieved articles applied to the title and abstract, and was performed by one author (AS). Subsequently, these studies were reviewed by a second author (AP) to determine that these studies met the appropriate inclusion criteria. Any uncertainties or 
disagreements were resolved after a consensus meeting with a third reviewer (MS). In the second phase, full texts for all of the above eligible studies were retrieved, and reviewed by AP and AS to ensure the selection criteria was maintained. Any disagreements were resolved through a third reviewer (KM), who was experienced with brain MRI research. Data extraction was performed by AS. The authors were all experienced in conducting systematic reviews.

\subsection{Inclusion and exclusion criteria}

Inclusion criteria for both aims ( $a$ and $b$ ):

i) Full text studies published in English prior to October 14, 2016.

ii) Studies involving adult humans

iii) Studies investigating the organisation and/or function of the brain using $f M R I$ and analysed using MVPA

iv) In press or accepted studies were also included during the hand search if authors were made aware of such.

For studies investigating musculoskeletal pain conditions (aim a), a healthy control comparison group was required. Studies investigating experimental pain (aim b) required methodology involving application of a noxious stimulation vs. a non-noxious control.

Exclusion criteria:

i) Studies including only participants with pain from medical conditions (i.e. cancer, autoimmune, surgical or visceral pain) or central nervous system pathologies (i.e. spinal cord injury or stroke)

ii) Reviews, systematic reviews, and meta-analyses.

\subsection{Outcomes}

The following items were systematically extracted from every article:

i) Publication details - authors, year of publication;

ii) Characteristics of trial (sample sizes, duration of symptoms, and pain scores);

iii) Patient characteristics (condition, gender and age);

iv) Imaging methods (including fMRI data acquisition and pre-processing, machine-learning) and outcomes, specifics of the investigative model such as type and location of stimulation, neuroimaging findings, results, and remarks;

v) Type of task performed in the scanner;

vi) Statistical approach, and

vii) Classification accuracy or associated reported functional outcome measures (e.g. behavioural results, pain scores).

\subsection{Risk of bias in individual studies}

In order to assess the methodological quality of each individual study, the STROBE statement and the Newcastle-Ottawa Scale (http://www.ohri.ca/programs/clinical epidemiology/oxford.asp) for cohort studies was utilized. The Cochrane Collaboration group recommends the Newcastle-Ottawa Scale as a quality assessment tool of observational studies (http://www.cochrane.org/). The Newcastle-Ottawa Scale consists of eight questions, distributed through three categories (selection, comparability, and outcome), with a total score of 10 points possible. Higher scores are indicative of greater methodological quality. As per a recent systematic review performed on MRI studies in chronic low back pain (Kregel et al., 2015), modifications were applied in the comparability category with studies earning one point when controlling for age or sex and another point for controlling for a 
minimum of two factors. Loss to follow-up was set at maximum $20 \%$. AS and AP independently applied the NOS to each study. Disagreements of ratings were discussed and final scores for each publication were determined at a consensus meeting with the third reviewer (MS), resulting in a final NOS assessment score. Given that the NOS scale is not specific to MRI studies, further methodological assessment was determined by the same authors applying the recently recommended guidelines for reporting an fMRI study (Poldrack et al., 2008). The authors were unable to locate similar criteria to evaluate structural MRI studies.

\subsection{Levels of Evidence}

The level of evidence for each study was assigned according to the NHMRC of Australia Evidence Hierarchy (https://www.nhmrc.gov.au/ files nhmrc/file/guidelines/developers/nhmrc levels grades evidence 120423.pdf). Studies were then grouped according to study methodologies employed to allow an overall strength of conclusion to be determined based on the NHMRC body of evidence matrix, accounting for the evidence base (study designs), consistency, clinical impact, generalisability and applicability. Evidence component A (Excellent) refers to a study with one or more level I studies (or several level II studies) with a low risk of bias that are consistent in their findings and possess a very large clinical impact as a result of the populations studied being in the same population as the target population.

Component B (Good) refers to one or two level II (or a systematic review/several level III) studies with a low risk of bias studies that are consistent in their findings (or inconsistencies can be explained). Clinical impact is substantial, with the population studied being similar to that of the target population. Component C (Satisfactory) studies consist of one or two level III studies with a low risk of bias, or level I or II studies with a moderate risk of bias with some inconsistency reflecting genuine uncertainty around the clinical question. Moderate clinical impact is evident, with the target population not necessarily being represented by the population studied. Component $D$ (Poor) studies do not fulfil the previous criteria.

\subsection{Preferred Reporting Items for Systematic Reviews}

This systematic review was performed in accordance with Strengthening the Reporting of Observational Studies in Epidemiology (STROBE) statement (The PLOS Medicine Editors, 2014).

\section{Results:}

\subsection{Study selection}

The search yielded 7634 results from all databases searched. After removing duplicates, 5457 articles were reviewed for eligibility, based on the established inclusion and exclusion criteria. After both screening phases, the reference lists of the 26 eligible records were checked as additional hand searches, 18 articles were included for review (Fig. 1). There was uncertainty between two reviewers (AS and AP) regarding inclusion eligibility for one study (based on title and abstract). This was resolved following a consensus meeting with the third reviewer (MS). Further uncertainty existed following full text revision for two studies. This was resolved by a third reviewer (KM). 


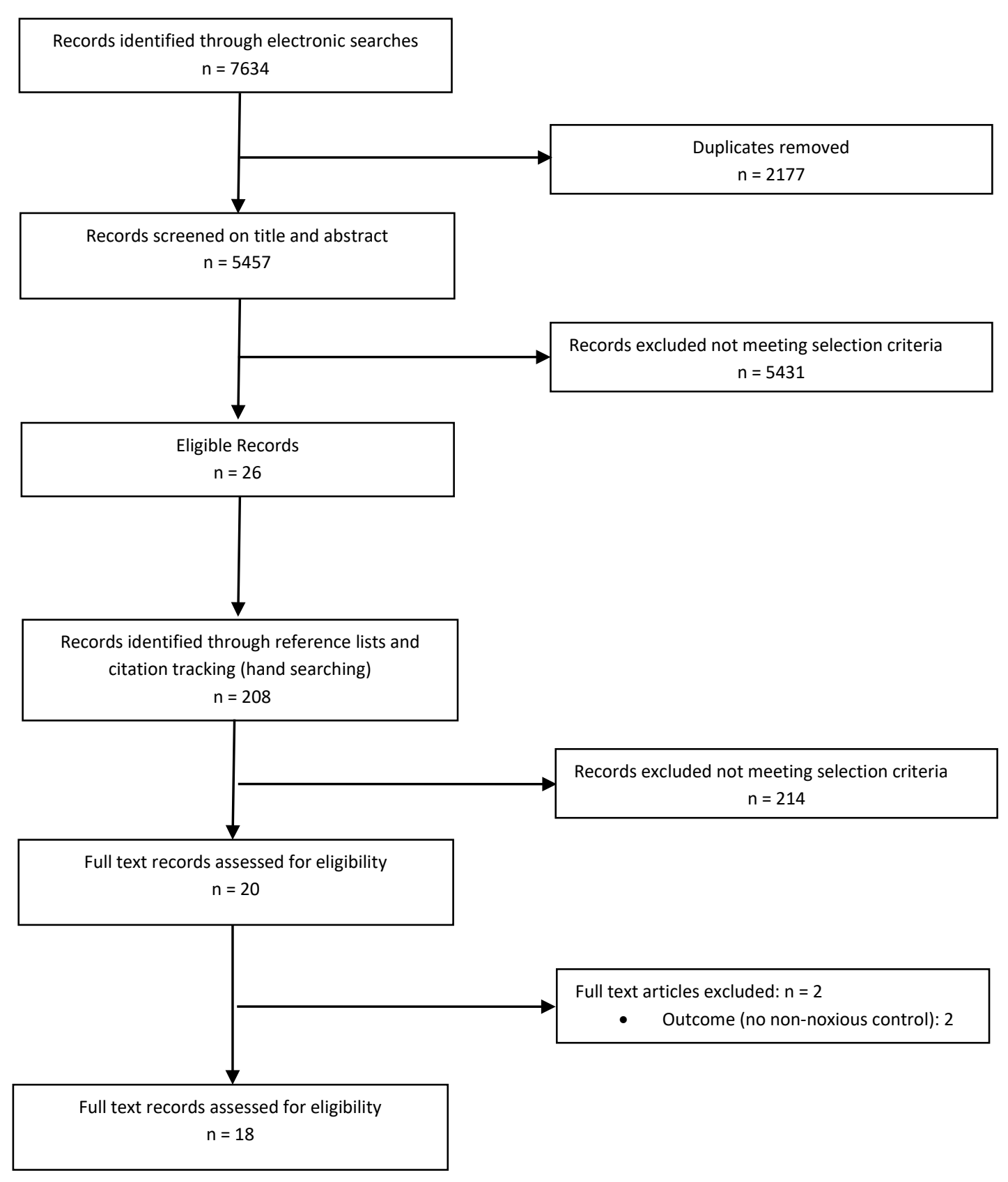

\subsection{Risk of bias}

Fig. 1: Study Selection Process

Table 2 demonstrates the results of the evaluation of methodological quality utilizing the NewcastleOttawa Scale. A total score of nine was possible, with one point awarded for fulfilment of each criteria, except criteria five, whereby two points was awarded if the study controlled for more than one factor. The results for fulfilling criteria associated with reporting an fMRI study (Poldrack et al., 2008) are shown in Supplementary Table 1, whilst the specific methodological details of the included studies are reported in Supplementary Table 2. Studies were performed utilizing various methods of MRI acquisition, pre-processing, analysis and prediction/ evaluation. In particular, machine-learning classifiers, feature selection and cross validation were highly variable between studies (Supplementary Table 2). Studies also varied in consideration of participant demographics (age, gender, handedness and race), with various noxious task stimuli utilized at differing anatomical locations. All studies were cross-sectional, resulting in the assigned level of evidence. 


\begin{tabular}{|c|c|c|c|c|c|c|c|c|c|c|}
\hline \multirow[b]{2}{*}{$\begin{array}{l}\text { First Author, Year of } \\
\text { Publication: }\end{array}$} & \multicolumn{10}{|c|}{ Newcastle-Ottawa Quality Assessment Scale } \\
\hline & 1 & 2 & 3 & 4 & 5 & 6 & 7 & 8 & $\begin{array}{r}\text { Total } \\
(/ 9) \\
\end{array}$ & $\begin{array}{l}\text { Level of } \\
\text { Evidence }\end{array}$ \\
\hline Baliki et al, 2011 & 1 & 1 & 1 & 1 & 1 & 1 & 1 & 1 & 8 & $\mathrm{III}-2$ \\
\hline Brodersen et al, 2012 & 0 & 1 & 1 & 1 & 2 & 1 & 1 & 1 & 8 & III-2 \\
\hline Brown et al, 2011 & 0 & 1 & 1 & 1 & 2 & 1 & 1 & 1 & 8 & III-2 \\
\hline Callan et al, 2014 & 1 & 0 & 1 & 1 & 2 & 1 & 1 & 1 & 8 & III-2 \\
\hline Cecchi et al, 2012 & 0 & 1 & 1 & 0 & 2 & 1 & 1 & 1 & 7 & III-2 \\
\hline Chang et al, 2015 & 0 & 1 & 1 & 1 & 0 & 1 & 1 & 1 & 6 & III-3 \\
\hline Corradi-Dell'Acqua et al, 2011 & 0 & 1 & 1 & 1 & 2 & 1 & 1 & 1 & 8 & III-2 \\
\hline Harper et al, 2016 & 1 & 1 & 1 & 1 & 2 & 1 & 1 & 1 & 9 & III-2 \\
\hline Krishnan et al, 2016 & 1 & 1 & 1 & 1 & 2 & 1 & 1 & 1 & 9 & III-2 \\
\hline Liang et al, 2013 & 0 & 1 & 1 & 0 & 2 & 1 & 1 & 1 & 7 & III-2 \\
\hline Lopez-Sola et al, 2016 & 1 & 0 & 1 & 1 & 2 & 1 & 1 & 1 & 8 & $\mathrm{III}-2$ \\
\hline Marquand et al, 2010 & 0 & 1 & 1 & 1 & 2 & 1 & 1 & 1 & 8 & III-2 \\
\hline Ritter et al, 2014 & 0 & 1 & 1 & 1 & 2 & 1 & 1 & 1 & 8 & III-2 \\
\hline Robinson et al, 2015 & 1 & 1 & 1 & 1 & 2 & 1 & 1 & 1 & 9 & III-2 \\
\hline Sundermann et al, 2014 & 1 & 0 & 1 & 1 & 1 & 1 & 1 & 1 & 7 & III-3 \\
\hline Ung et al, 2014 & 1 & 1 & 1 & 1 & 2 & 1 & 1 & 1 & 9 & III-2 \\
\hline Wager et al, 2013 & 0 & 1 & 1 & 1 & 2 & 1 & 1 & 1 & 8 & III-2 \\
\hline Woo et al, 2015 & 0 & 1 & 1 & 1 & 2 & 1 & 1 & 1 & 8 & III-2 \\
\hline
\end{tabular}

Table 2: Quality Assessment of Individual Studies

\section{Quality Assessment Criteria Questions:}

1. Representativeness of the exposed cohort

2. Selection of the non-exposed cohort

3. Ascertainment of exposure

4. Demonstration that outcome of interest was not present at start of study

5. Comparability of cohorts on the basis of the analysis

6. Assessment of outcome

7. Was follow-up long enough for outcomes to occur

8. Adequacy of follow up of cohorts

Seven studies investigated patient conditions with comparative controls (Baliki, Schnitzer, Bauer, \& Apkarian, 2011; Callan, Mills, Nott, England, \& England, 2014; Harper, Shah, Icheso, Gerstner, \& Peltier, 2016; Lopez-Sola et al., 2016; Robinson et al., 2015; Sundermann et al., 2014; Ung et al., 2014) and reported results on the ability of machine-learning algorithms to differentiate patient groups from healthy control participants.

Eleven studies utilized healthy control participants (using them as their own controls), during experimental pain paradigms aimed to investigate the ability of machine-learning to differentiate individuals stimulated with noxious stimuli from those stimulated with non-noxious stimuli. One study utilized retrospective data collection (Sundermann et al., 2014).

There was excellent agreement between the two raters on both the Newcastle-Ottawa Scale quality assessment criteria questions $(134 / 144 ; 93.1 \%)$ and guidelines for reporting an fMRI study $(219 / 225$; 97.3\%). In the majority of studies, the 'Selection' criteria demonstrated low scores, as represented by an incomplete description of the representativeness of the investigated sample. All studies (apart 
from Chang et al. (L. J. Chang, Gianaros, Manuck, Krishnan, \& Wager, 2015)) scored 7/9 or greater on the Newcastle-Ottawa Scale outcome measure criteria.

In so far as fulfilling criteria for reporting an fMRI studies (Supplementary Table 1), five studies (35.7\%) failed to specify their experimental design and five studies did not provide details of the planned comparisons. This tool also has a section on behavioural outcome/psychological measures. Seven studies did not report the results. Four studies did not report details for statistical thresholding. The remainder of the other evaluated criteria (human subject details and ethical approval; data acquisition, data pre-processing and statistical modelling details) were fulfilled by all studies.

\subsection{Study characteristics}

Table 3 shows the participant characteristics for each individual study. The study sample (patients and controls), imaging parameters (magnet strength, acquisition and pre-processing methodology), machine-learning parameters, main findings and remarks are outlined.

In the studies involving patient groups, three investigated chronic low back pain (Baliki et al., 2011; Callan et al., 2014; Ung et al., 2014), three investigated fibromyalgia (Lopez-Sola et al., 2016; Robinson et al., 2015; Sundermann et al., 2014) and one study each investigated temporomandibular disorder (Harper et al., 2016) and knee osteoarthritis (Baliki et al., 2011). Sample sizes in these studies ranged from $10-47$. Three studies investigated female participants only (LopezSola et al., 2016; Robinson et al., 2015; Sundermann et al., 2014). There were variable gender participation rates in the remaining studies (20-86\% females). Overall, the pooled mean age of the patient groups investigated was 45.1 (SD: 9.1) years.

In the healthy sample studies, participant numbers were generally small (range: $6-28$ ), although one study investigated four different healthy samples of between $20-33$ individuals, with a total of 114 participants involved (T. D. Wager et al., 2013); and another study included a comparative group of 183 participants (L. J. Chang et al., 2015). One study investigated female participants only (Corradi-Dell'Acqua, Hofstetter, \& Vuilleumier, 2011) and one study investigated male participants only (Marquand et al., 2010). The remaining studies had variable gender participation rates (20-69\% females). The average age for the combined study group participants was 32.7 (7.6) years. 


\section{STRUCTURAL}

\section{Author/ \\ Study Population: Image Acquisition:}

Classification Base:

Baliki et al. (2011)

Whole-brain barcode ('brain signature') based on grey matter density

CLBP: 36 ( 23 male)
age $-48.2 \pm 11.4$ yrs
CRPS: 28 (4 male)
age $-40.6 \pm 7.4$ yrs
Knee OA: 20 (16 male)
age $53.5 \pm 7.4$ yrs
HC: 46 ( 20 male)
age $-38.8 \pm 12.5$ yrs

All right-handed

$256 \mathrm{~mm}$

\section{Main Findings (Accuracy in Remarks:}

\section{discriminating patients from} controls):

3 T T1W

TE: $3.36 \mathrm{~ms}$; TR: $2500 \mathrm{~ms} ; 1 \times 1 \mathrm{x}$

$1 \mathrm{~mm}^{3}$ voxel size; flip angle $=9^{\circ}$; $\quad$ cLBP.

in-plane matrix resolution, $256 x \quad \mathrm{Sp}=94 \% \quad \mathrm{Sp}=91 \%$

256; slices, 160 ; field of view, $\quad$ Se $=81 \%$.

$\mathrm{Sp}=91 \%$
$\mathrm{Se}=73 \%$
Somatosensory, insular and motor regions demonstrated significant decreased GM volume for $>5$ yrs duration of pain.

\section{Ung et al. (2014)}

Grey matter density
cLBP: 47 (25 male)

age $-37.3 \pm 12.2$ yrs

Mean pain duration -8.6

\pm 7.8 yrs

HC: 47 (25 male)

age $-37.7 \pm 7.8$ yrs

\section{T T1W}

3D inversion recovery fast spoiled gradient-recalled pulse sequence (3D IR-FSPGR)

TE: $1.71 \mathrm{~ms}$; TR: $8.18 \mathrm{~s} ; 1.5 \mathrm{~mm}$ slice thickness; $0.86 \times 0.86 \mathrm{~mm}^{2}$ voxel size, 124 slices

Magnet size not reported

MPRAGE

age -44.1 yrs

HC: 12 age- and sex-

matched

age -42.2 yrs
TE: 3.7ms; TR: $8.1 \mathrm{~ms} ; 1 \times 1 \times 1$ $\mathrm{mm}^{3}$ voxel size; matrix $=256 \mathrm{x}$ $256 \times 170 \mathrm{~mm}$; field of view $=$ $240 \times 240 \times 170 \mathrm{~mm}$; flip angle $=$ $8^{\circ}$
Different distributed patterns of GM density between groups:

Average accuracy $=76 \%$.

$\mathrm{Se}=76 \%$

$\mathrm{Sp}=75 \%$

$\mathrm{PPV}=75 \%$

$\mathrm{NPV}=75 \%$

Accuracy rates in discriminating

\section{patients from controls:}

Logistic - 64\%

MLP - 69\%

SVM $-72 \%$

IB3 - 53\%

148 classifier - 76\%

Naïve Bayes - 64\%; SVM \& J48

outperformed base logistic rate
Decreased GM density adjacent to the right amygdala, left medial orbital gyrus, right V2.

Increased GM density right cerebellum regions of the temporal lobe, left S1 and S2, left $\mathrm{M} 1$, right $\mathrm{V} 1$, and right dIPFC

Self-reported pain intensity outperformed the neuroimaging data set for peak and mean accuracy across all 6 learning classifiers.

Left amygdala volume was the most predictive neuroimaging feature for classifying individuals, whereas anger was the most predictive self-report feature 


\begin{tabular}{|c|c|c|c|c|}
\hline \multicolumn{5}{|c|}{ FUNCTIONAL: Patient Populations } \\
\hline $\begin{array}{l}\text { Author/ } \\
\text { Applied Stimuli: }\end{array}$ & Study Population: & Image Acquisition: & $\begin{array}{l}\text { Main Findings (Accuracy in } \\
\text { discriminating patients from } \\
\text { controls): }\end{array}$ & Remarks: \\
\hline Callan et al. (2014): & $\begin{array}{l}\text { cLBP: } 13 \text { ( } 4 \text { male) } \\
\text { age }-51.8 \text { yrs (43-65yo) }\end{array}$ & $\begin{array}{l}1.5 \mathrm{~T} \\
\mathrm{~T} 2 *-\mathrm{W} \text { EPI }\end{array}$ & $\begin{array}{l}92 \% \text { correct classification for both the } \\
\text { chronic pain group (Se) and the }\end{array}$ & $\begin{array}{l}\text { Classification accuracy determined by } \\
\text { recognition of MVPA in left S1; (lower activity }\end{array}$ \\
\hline $\begin{array}{l}\text { Electrical stimulation } \\
\text { applied to lumbar spine } \\
\text { at tolerance }\end{array}$ & $\begin{array}{l}\text { HC: } 13 \text { ( } 4 \text { male) } \\
\text { age }-48.7 \text { yrs (38-62yo) } \\
\text { Matched for handedness } \\
\text { (10 right-handed), race } \\
\text { (10 Caucasion, } 3 \text { Black } \\
\text { African Americans) }\end{array}$ & $\begin{array}{l}\text { TE: } 60 \mathrm{~ms} ; \text { TR: } 3670 \mathrm{~ms} ; \text { flip angle } \\
=90^{\circ} ; 3 \times 3 \times 3 \mathrm{~mm}^{3} \text { voxel } \\
\text { resolution; } 36 \text { slices. }\end{array}$ & normal group (Sp) & $\begin{array}{l}\text { in chronic pain group) and inferior parietal } \\
\text { cortex (higher activity) }\end{array}$ \\
\hline Harper et al. (2016): & $\begin{array}{l}\text { TMD: } 10 \text { (1 male) } \\
\text { age }-24.9 \pm 1.2 \text { yrs }\end{array}$ & $\begin{array}{l}3 \mathrm{~T} \\
\mathrm{~T} 2 *-\mathrm{W} \text { spiral sequence }\end{array}$ & $\begin{array}{l}\text { Average prediction accuracy of TMD } \\
\text { patients vs } \mathrm{HC}=60 \% \text { (NS) }\end{array}$ & \\
\hline $\begin{array}{l}\text { Pressure stimulation } \\
\text { applied to left temporalis } \\
\text { and left thumb regions at } \\
\text { medium and high pain } \\
\text { intensities }\end{array}$ & $\begin{array}{l}\text { HC: } 10 \text { (1 male) } \\
\text { age }-26.9 \pm 4.4 \text { yrs } \\
\text { Ethnicity and age } \\
\text { matched }\end{array}$ & $\begin{array}{l}\text { TE: } 30 \mathrm{~ms} \text {; TR: } 2.5 \mathrm{~s} \text {; flip angle = } \\
90^{\circ} ; \text { matrix size } 64 \times 64 \mathrm{~mm} \\
\text { with } 48 \text { slices, field of view = } \\
220 \mathrm{~mm} \text {, and } 3.44 \times 3.44 \times 3 \\
\text { mm voxels }\end{array}$ & & \\
\hline Lopez-Sola et al. (2016): & $\begin{array}{l}\text { FMS: } 37 \text { Women } \\
\text { age }-46.3 \pm 7.8 \text { yrs }\end{array}$ & $\begin{array}{l}3 \text { T with 8-channel phased-array } \\
\text { head coil and single-shot EPI }\end{array}$ & $\frac{\text { NPS response: }}{\text { Using Positive Regions: }}$ & $\begin{array}{l}\text { FM-pain and multi-sensory classifiers could } \\
\text { accurately classify patients vs controls using }\end{array}$ \\
\hline $\begin{array}{l}\text { Pressure (low and severe } \\
\text { intensities) applied to } \\
\text { right thumbnail. }\end{array}$ & $\begin{array}{l}\text { HC: } 35 \text { women } \\
\text { age }-43.9 \pm 6.1 \text { yrs }\end{array}$ & $\begin{array}{l}\text { TE: } 35 \mathrm{~ms} ; \text { TR: } 2.0 \mathrm{~ms} \text {; flip angle } \\
=90^{\circ} ; \text { field of view }=230 \mathrm{~mm}, 96 \\
\text { X 69-pixel matrix, and slice }\end{array}$ & $\begin{array}{l}\text { FMS vs } \mathrm{HC}=69 \% \text { (SE: } 6 \% \text { ) } \\
\text { Using Negative Regions: } \\
\text { FMS vs } \mathrm{HC}=71 \% \text { (SE: } 5 \% \text { ) }\end{array}$ & $\begin{array}{l}\text { images from either task indicating that the } \\
\text { two classifiers might reflect a generalized } \\
\text { brain reorganization in FM }\end{array}$ \\
\hline $\begin{array}{l}\text { Multisensory stimuli } \\
\text { involved concurrent } \\
\text { visual, auditory and } \\
\text { tactile-motor stimulation }\end{array}$ & $\begin{array}{l}\text { Illness duration: } 80+/-52 \\
\text { months. Participants } \\
\text { matched for age, } \\
\text { education and } \\
\text { handedness }\end{array}$ & $\begin{array}{l}\text { thickness of } 4 \mathrm{~mm} \text { (inter-slice } \\
\text { gap, } 1 \mathrm{~mm} \text { ); } 22 \text { slices }\end{array}$ & $\begin{array}{l}\text { FM-pain classifier response: } \\
\text { FMS vs HC = 70\% (SE: } 5 \%) \\
\text { Se }=74 \%(95 \% \mathrm{Cl}: 62-86) \\
\text { Sp }=66 \%(95 \% \mathrm{Cl}: 53-79) \\
\text { Multi-sensory classifier response: } \\
\text { FMS vs HC }=90 \% \text { (SE: } 4 \%)\end{array}$ & \\
\hline
\end{tabular}


$\mathrm{Se}=84 \%(95 \% \mathrm{Cl}: 73-93)$

$\mathrm{Sp}=94 \%(95 \% \mathrm{Cl}: 87-100)$

Combined response:

FMS vs $\mathrm{HC}=93 \%$ (SE: $3 \%$ )

$\mathrm{Se}=92 \%(95 \% \mathrm{Cl}: 84-98)$

$\mathrm{Sp}=94 \%(95 \% \mathrm{Cl}: 87-100)$

\begin{tabular}{lll}
\hline Sundermann et al. (2014): & FMS: 17 Women & $3 \mathrm{~T}$ \\
& age $-52.6 \pm 7.9 \mathrm{yrs}$ & T2*-W EPI \\
Resting State Functional & RA: 16 Women & TE: $35 \mathrm{~ms} ; \mathrm{TR}: 5000 \mathrm{~ms}$; matrix \\
Connectivity with & age $-47.7 \pm 6.5 \mathrm{yrs}$ & dimensions $=64 \times 64$, field of \\
'experimental pain & HC: 17 Women & view $=210 \mathrm{~mm}, 36$ transversal \\
stimulus' & age $-49.5 \pm 8.9 \mathrm{yrs}$ & $\begin{array}{l}\text { slices, scan time }=3.4 \text { secs, slice } \\
\text { thickness }=3.6 \mathrm{~mm} \text {, pixel size }= \\
\end{array}$ \\
& & $3.6 \times 3.6 \mathrm{~mm}^{2}$
\end{tabular}

Not significantly able to differentiate

FM or RA patients from $\mathrm{HC}$

FM vs HC accuracy $=62 \%$ (NS).

$\mathrm{RA}$ vs $\mathrm{HC}=58 \%$ (NS)
Some indirect post-hoc evidence in support of the feasibility of approach with best classification accuracy (79\%) achieved by the nearest neighbour model followed by different SVM models.

\begin{tabular}{|c|c|c|c|c|}
\hline \multicolumn{5}{|c|}{ FUNCTIONAL: Healthy Participants } \\
\hline $\begin{array}{l}\text { Author/ } \\
\text { Applied Stimuli: }\end{array}$ & Study Population: & Imaging Acquisition: & $\begin{array}{l}\text { Main Findings (Accuracy in } \\
\text { discriminating noxious from non- } \\
\text { noxious stimuli): }\end{array}$ & Remarks: \\
\hline $\begin{array}{l}\text { Brodersen et al. (2012): } \\
\text { Near-threshold laser } \\
\text { stimulus at individually } \\
\text { calibrated pain-detection } \\
\text { threshold }\end{array}$ & $\begin{array}{l}\text { HC: } 16 \text { ( } 5 \text { male }) \\
\text { age range }=19-30 \text { yrs }\end{array}$ & $\begin{array}{l}3 \mathrm{~T} \\
\text { T2*-W EPI } \\
\text { TE: 30ms; TR: 3s; flip angle = } \\
90^{\circ} \text {; matrix } 64 \text { X 64; field of view } \\
192 \mathrm{~mm} \times 192 \mathrm{~mm} ; 41 \text { axial } \\
\text { slices; slice thickness } 3 \mathrm{~mm}\end{array}$ & $\begin{array}{l}\text { Whole-brain activity before ( } 58 \%) \text { and } \\
\text { during }(61 \%) \text { application of near- } \\
\text { threshold laser stimulus }\end{array}$ & $\begin{array}{l}\text { Combined activity in classical pain processing } \\
\text { regions provided the most accurate } \\
\text { prediction of pain perception }\end{array}$ \\
\hline $\begin{array}{l}\text { Brown et al. (2011): } \\
\text { Painful thermal stimulus } \\
\text { (applied to left forearm) }\end{array}$ & $\begin{array}{l}\frac{\mathrm{HC}:}{\text { age }}-22.7 \pm 3.6 \text { yrs } \\
\text { Validation Confirmation: } \\
\left.\frac{\mathrm{HC}:}{\text { age }}-25 \text { male }\right) \\
\text { yrs }\end{array}$ & $\begin{array}{l}3 \text { T with 8-channel receiver-only } \\
\text { phased-array head coil } \\
\text { T2*-W gradient spiral in/out } \\
\text { pulse sequence TE: } 20 \mathrm{~ms} ; \mathrm{TR} \text { : } \\
2000 \mathrm{~ms} \text {; flip angle }=77^{\circ}, 64 X 64 \\
\text { matrix, and } 30,4 \mathrm{mmm}\end{array}$ & $\begin{array}{l}\text { Study 1: } \\
\text { Average accuracy }=87 \% \\
\text { PPV }=90 \% \\
\text { NPV = 85\%; } \\
\text { Study 2: Confirmatory Group } 75 \% \\
\text { PPV }=84 \%\end{array}$ & $\begin{array}{l}\text { Pain stimulus influenced by greater BOLD } \\
\text { signal in the bilateral mid-INS, bilateral S2, } \\
\text { contralateral posterior INS, contralateral S1 } \\
\text { cortex and contralateral M1. } \\
\text { Non-painful stimulus was influenced by }\end{array}$ \\
\hline
\end{tabular}


interleaved slices parallel to the NPV $=74 \%$ intercommisural line

Cecchi et al. (2012):

Thermal stimuli applied to back region whilst continuously rating perceived pain intensity

$3 \mathrm{~T}$

$$
\text { age }-35.2 \pm 11.5 \text { yrs }
$$$$
\text { HC: } 14 \text { (7 male) }
$$

Right-handed

T2*-W EPI

TE: 30ms; TR: 2500ms; flip angle $=90^{\circ}$; slice thickness $=3 \mathrm{~mm}$, inplane resolution $=64 \times 64 ; 36$ slices greater BOLD signal in the bilateral M1 and bilateral pregenual cingulate cortex.

\begin{tabular}{|c|c|}
\hline \multirow[t]{2}{*}{ Chang et al. (2015): } & HC: 30 (60\% male) \\
\hline & age $-25.2 \pm 7.4$ yrs \\
\hline $\begin{array}{l}\text { Thermal stimulus applied } \\
\text { to volar surface of left }\end{array}$ & $\begin{array}{l}\text { subjected to thermal } \\
\text { stimulus. }\end{array}$ \\
\hline forearm and dorsal & In a separate sample, \\
\hline surface of left foot & HC: 183 (48\% male) \\
\hline & $\overline{\text { age }}-42.8 \pm 7.3$ yrs \\
\hline & $\begin{array}{l}\text { exposed to aversive } \\
\text { images }\end{array}$ \\
\hline
\end{tabular}

$75 \%$ combined accuracy with

correlating dynamic heat pain with

combined model incorporating

psychophysics and brain physiology

$\begin{array}{ll}3 \mathrm{~T} & \text { Pain Classifier: } \\ \text { EPI } & \text { Pain High vs. Low: }\end{array}$

TE: $25 \mathrm{~ms} ;$ TR: $1300 \mathrm{~ms} ;$ matrix $=$ Accuracy $=82 \%$ (SE: 6\%)

$64 \times 64$; flip angle $=90^{\circ} ; 34 \quad$ Pain vs. Emotion:

$3 \mathrm{~mm}$ slices with no gap

Accuracy $=89 \%$ (SE: 4\%)

Highly Aversive vs. Non Aversive:

Accuracy $=28 \%$ (SE: $5 \%$ )

PINES Classifier:

Pain High vs. Low:

Accuracy $=61 \%$ (SE: $8 \%$ )

Pain vs. Emotion:

Accuracy $=7 \%$ (SE: 3\%)

Highly Aversive vs. Non Aversive:

Accuracy $=94 \%$ (SE: 3\%)

Corradi-Dell-Acqua et al
(2011):
Thermal stimulation to
right palm

\section{$\mathrm{HC}: 28$ female age range $=19-31$ yrs}

\section{$3 \mathrm{~T}$}

$\mathrm{T} 2 *-\mathrm{W}$

TE: 30ms; TR: 2100ms; flip angle $=90^{\circ}$; in-plane resolution of 64

$X 64$ voxels (voxel size, $3 \times 3$ $\mathrm{mm}$ ); 32 slices; slice thickness =
The right alNS demonstrated shared

neural activity in both 'felt' and 'seen'

pain. Right and left alNS shared

patterns of brain activity between

'seen' pain and 'felt' pain 


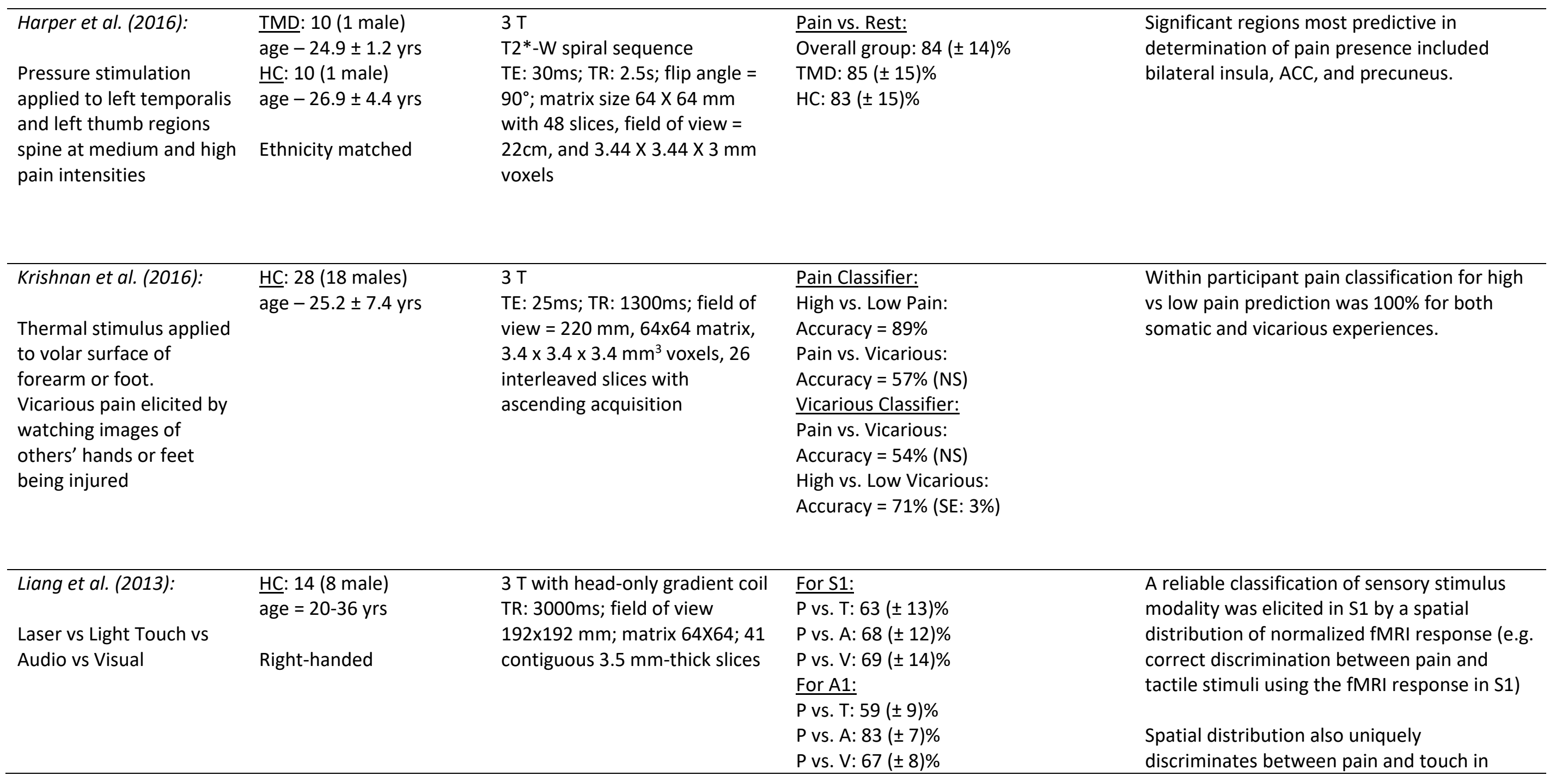


Marquand et al. (2010):

Thermal stimulus to volar surface of right forearm

$$
\begin{aligned}
& \underline{\mathrm{HC}}: 15 \text { (15 male) } \\
& \text { age }-27.9 \pm 7.3 \mathrm{yrs}
\end{aligned}
$$

Right- handed
$3 \mathrm{~T}$

T2*-W EPI

TE: 40ms; TR: $3000 \mathrm{~ms}$; flip angle $=90^{\circ}$; field of view $=24 \mathrm{~cm}$; matrix size $64 \times 64$; in-plane resolution $3.75 \times 3.75$; slice thickness $=3 \mathrm{~mm}$ with $0.3 \mathrm{~mm}$ gap between slices
Thermal Detection vs Pain Detection:

Thermal Detection vs Pain Tolerance

Threshold:

$\mathrm{GC}=92 \%$; RVM = 86\%; SVM = 91\%;

Pain Detection vs Pain Tolerance

Threshold:

$\mathrm{GC}=72 \% ; \mathrm{RVM}=69 \% ; \mathrm{SVM}=68 \%$

Ritter et al. (2014):
Individualized heat pain
thresholds (60\% VAS
maximum) were provided
to left inner forearm or
lateral calf region

HC: 15 (12 male) age -24.6 yrs, range 1932 yrs

Right-handed

to left inner forearm
lateral calf region

\section{T with 32-channel head coil} T2*-W EPI

TE: 26ms; TR: 1970ms; 2X2X2 $\mathrm{mm}^{3}$ voxel resolution; parallel imaging, 33 slices, 25\% gap; field of view $=208 \mathrm{~mm}$ covering area between brainstem and S2

\section{During painful stimulation: \\ rACC $=88 \pm 9 \%$ \\ Left dIPFC $=79 \pm 13 \%$}

Right dIPFC $=83 \pm 12 \%$

Parietal operculum $=91 \pm 9 \%$

\section{During Anticipation:}

$\mathrm{rACC}=90 \pm 7 \%$

Left dIPFC $=74 \pm 17 \%$

Right dIPFC $=79 \pm 12 \%$

Parietal operculum $=84 \pm 8 \%$

Wager et al. (2013):
Study 1: Thermal stimulus
on left forearm at 4
different intensities -
innocuous warmth (level
1), or 3 levels of painful

$\begin{array}{ll}\text { Study 1: } & \text { Studies 1 \& 4: } \\ \text { HC: } 20 \text { (12 male) } & 1.5 \text { T } \\ \text { age }=28.8 \pm 7.5 \text { yrs } & \text { EPI } \\ \text { Study 2: } & \text { TE: 34ms; TR: 2000ms; } \\ \text { HC: 33 (11 male) } & \text { Study 2: } \\ \text { age }=27.9 \pm 9.0 \text { yrs } & 3 \text { T } \\ \text { Study 3: } & \text { EPI }\end{array}$

\section{Study 1:}

Discrimination of pain $\left(>45^{\circ} \mathrm{C}\right)$ from no pain $\left(<45^{\circ} \mathrm{C}\right)$ or pain vs anticipation or pain vs recall:

Se $\geq 94 \%$; Sp $\geq 94 \%$.

Study 2:

Pain vs Warmth:
No greater than chance finding for white matter or control regions or interstimulus interval 
heat $(3,5$, and 7$)$

Study 2: Thermal stimulus on left forearm across 6 temps $-44.3^{\circ} \mathrm{C}-49.3^{\circ} \mathrm{C}$ Study 3: noxious heat $\left(46.6+/-1.7^{\circ} \mathrm{C}\right)$, warmth $\left.\left(39.9+/-2.8^{\circ} \mathrm{C}\right)\right)$ and viewed images related to social pain (rejecter vs friend)

Study 4: 2 IV infusions of remifentanil (opioid agonist) whilst delivering $\mathrm{X} 18$ warm trials and X18 pain trials
HC: 40 (19 male)

age $=20.8 \pm 2.6 \mathrm{yrs}$

Study 4:

HC: 21 (10 male)

age $=24.7 \pm 4.2 \mathrm{yrs}$
TE: 20ms; TR: $2000 \mathrm{~ms}$;

\section{Study 3:}

$1.5 \mathrm{~T}$

$\mathrm{T} 2 *$-W spiral in out sequence

TE: 40ms; TR: $2000 \mathrm{~ms}$
$\mathrm{Se}=93 \%$ (95\%Cl: 84-100)

$\mathrm{Sp}=93 \%(95 \% \mathrm{Cl}: 84-100)$;

Study 3:

Pain vs Warmth:

$\mathrm{Se}=85 \%$ (95\%Cl: 76-94)

$\mathrm{Sp}=78 \%$ (95\%Cl: 67-89)

Pain vs Rejector:

$\mathrm{Se}=85 \%(95 \% \mathrm{Cl}: 76-94)$

$\mathrm{Sp}=73 \%(95 \% \mathrm{Cl}: 61-84)$

Study 4:

Pain vs Warmth (before drug

treatment):

$\mathrm{Se}=90 \%(95 \% \mathrm{Cl}: 79-100)$

$\mathrm{Sp}=81 \%$ (95\%Cl: 65-95)

(during drug treatment):

$\mathrm{Se}=86 \%$ (95\%Cl: 73-96)

$\mathrm{Sp}=62 \%(95 \% \mathrm{Cl}: 42-84)$ contralateral specificity in S1 and S2

\begin{tabular}{lll}
\hline Woo et al. (2015): & HC: 60 (29 male) recently & $1.5 \mathrm{~T}$ \\
& experienced an unwanted & T2*-W spiral in-out sequence \\
Thermal stimulation to & romantic relationship & TE: $40 \mathrm{~ms} ;$ TR: $2000 \mathrm{~ms}$; flip angle \\
left forearm & break-up within the past & $=84^{\circ} ;$ filed of view $=22 \mathrm{~cm}, 24$ \\
& 6 months, and indicated & axial slices $; 3.5 \times 3.5 \times 4.5 \mathrm{~mm}^{3}$ \\
& that they felt rejected & voxels \\
& when thinking about their & \\
& break-up & \\
& age $=20.8 \pm 3.0$ yrs &
\end{tabular}

\section{Pain Classifier:}

Heat-pain vs Ex-partner:

Accuracy $=100 \%(95 \% \mathrm{Cl}: 100-100)$

Heat-pain versus Warmth:

Accuracy $=92 \%$ (95\%Cl: 84-99)

Ex-partner vs Friend:

Accuracy $=59 \%(95 \% \mathrm{Cl}: 47-72 ; \mathrm{NS})$

Rejection Classifier:

Ex-partner vs Heat-pain:

Accuracy $=88 \%$ (95\%Cl: 80-96)

Ex-partner vs Friend:

Accuracy $=80 \%$ (95\%Cl: 69-90)

Heat-pain versus Warmth:

Accuracy $=59 \%(95 \% \mathrm{Cl}: 47-72 ; \mathrm{NS})$
Heat-pain versus Warmth was predicted by activation in many regions associated with nociceptive processing and endogenous pain control, such as right (contralat) dpINS, bilateral S2, medial thalamus and PAG.

Pain and rejection-related patterns included significantly different weights in dpINS, thalamus and PAG, but not $\mathrm{dACC}$, aINS and S2 


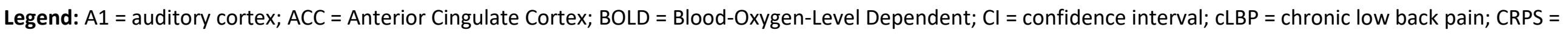

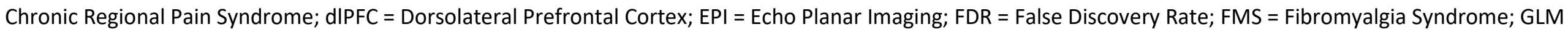

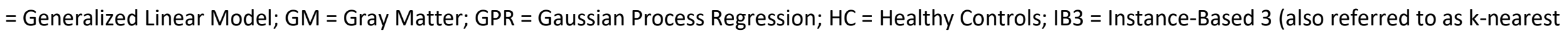
neighbors); INS = insula; LOO-CV = Leave-One-Out-Cross-Validation; LASSO-PCR = Least Absolute Shrinkage and Selection Operator and Principle Components Regression (LASSO-PCR); M1 = Primary Motor Cortex; MLP = Multilayer Perceptron Classifier; MVPA = Multivariate Pattern Analysis; NPS = Neurological Pain

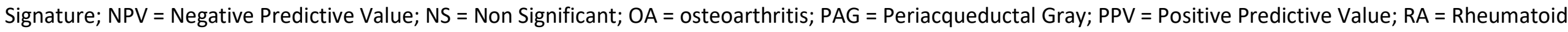

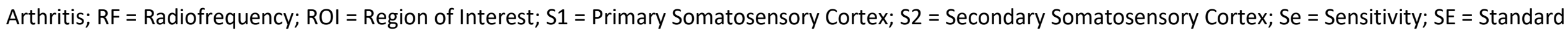
Error; SLR = Sparse Logistic Regression; Sp = Specificity; SPM = Statistical Parametric Mapping; SVM = Support Vector Machine; T = Tesla; TE = Echo Time; TR = Repetition Time; TMD = Temporomandibular Disorder; V1 = Primary Visual Cortex; V2 = Secondary Visual Cortex; VAS = Visual Analogue Scale; W = Weighted

Table 3: Evidence table of structural and functional brain MRI in individuals with musculoskeletal pain and/or healthy controls 


\subsection{Discriminating painful conditions from healthy controls:}

\subsubsection{Structural MRI}

Three studies investigated the ability to classify patients from controls via T1-weighted MRI (Baliki et al., 2011; Robinson et al., 2015; Ung et al., 2014). Each study investigated different patient groups (chronic low back pain vs knee osteoarthritis vs healthy control participants (Baliki et al., 2011); chronic low back pain vs healthy controls (Ung et al., 2014); fibromyalgia vs healthy controls (Robinson et al., 2015)) and utilized varying MRI parameters (grey matter density or regional brain volumes) and machine-learning techniques in an attempt to accurately classify patients from healthy controls on the basis of structural changes in the brain. The studies also investigated different aims, with one set of studies investigating participant within-group classification (Baliki et al., 2011); one investigating between-group classification (Ung et al., 2014), whilst a further study compared patient and healthy control findings in MRI against mood and pain classification accuracies (Robinson et al., 2015).

One study used support vector machine analysis to investigate a whole-brain pattern of grey matter density to discriminate between unmedicated, non-neuropathic chronic low back pain patients and healthy controls (Ung et al., 2014). The mean accuracy obtained was $76 \%$ (sensitivity $=76 \%$; specificity $=75 \%$; positive predictive value $=75 \%$; negative predictive value $=76 \%$ ). The support vector machine classifier identified pathological changes distributed across the brain with variable grey matter density changes. Regions that discriminated chronic low back pain individuals from healthy controls included increased grey matter density in the right cerebellum, temporal lobe regions, left primary and secondary somatosensory cortices (S1 and S2), left primary motor cortex (M1), right calcarine sulcus (primary visual cortex; V1) and the right dorsolateral prefrontal cortex; whilst reduced grey matter density was demonstrated adjacent to the right amygdala, left medial orbital gyrus and right cuneus (secondary visual cortex; V2).

Another study investigating chronic low back pain utilized individual subject whole-brain barcodes, which were generated using corrected grey matter density across 82 brain region of interests (Baliki et al., 2011). These were compared against group average normalized grey matter density for various patient conditions and healthy controls. Correctly identifying individual subjects to their respective group based on the maximum correlation of each barcode with group barcodes was high for specificity (healthy: $86.2 \%$, chronic low back pain: $94.2 \%$ and knee osteoarthritis: $90.9 \%$ ) and sensitivity (healthy: $80 \%$, chronic low back pain: $81.2 \%$, and knee osteoarthritis: $72.6 \%$ ). Different pain conditions demonstrated different regional increases or decreases in grey matter density. Secondary somatosensory cortices, bilateral insulae and dorsal and orbital frontal regions, besides the hippocampus were those that better differentiated between groups. It was not reported which combination or grey matter density across the 82 brain regions of interest differentiated between individual conditions and healthy controls.

One study investigated whether brain imaging, when combined with various machine-learning classifiers was able to outperform self-reported pain intensity, when predicting the presence of fibromyalgia or healthy controls (Robinson et al., 2015). Structural volumes from 55 brain regions were derived from MRI. Common machine-learning algorithms estimated various models representing brain volumes, pain and mood ratings. Support vector machines (72.2\%) and $\mathbf{J 4 8}$ decision tree $(75.5 \%)$ machine-learning classifiers successfully outperformed the base logistic rate for discriminating between fibromyalgia and healthy control participants. Among neuroimaging features, bilateral reductions in cerebellar cortex and thalamus, reduced left amygdala, accumbens 
and right pallidum volumes were demonstrated in the fibromyalgia group when compared to the healthy controls.

There is Level III-2 evidence that various machine-learning algorithms when combined with structural MRI may distinguish between patient groups and healthy controls (Grade of recommendation: $\mathrm{C})$.

\subsubsection{Functional MRI}

\subsubsection{Resting State Functional Connectivity}

One study investigated the ability of resting state functional connectivity differences in the default mode network and salience network to distinguish between fibromyalgia and healthy control participants, using retrospective baseline data from previously performed studies (Sundermann et al., 2014). Functional connectivity features in terms of pairwise region of interest correlation coefficients within the salience network and default mode network were used to calculate classification accuracies via various machine-learning models. No models were able to distinguish between fibromyalgia and healthy controls (61.8\%, not significant).

There is limited Level III-3 evidence that various machine-learning algorithms with model optimization cannot distinguish between fibromyalgia and healthy control participants when measuring resting state functional connectivity differences in the default mode network and salience network (Grade of recommendation: D).

\subsubsection{Functional MRI and Evoked Tasks}

One study investigated the use of electrical stimulation being applied to the lumbar spine region at individual pain tolerance levels to discriminate between groups of participants with chronic low back pain and healthy control participants (Callan et al., 2014). Investigators analysed contrast fMRI images in stimulation versus rest (no stimulation) in "pain-related brain regions" (Giesecke et al., 2004) (somatosensory cortices: S1, S2, inferior parietal cortex, insula and anterior cingulate cortex). The overall classification accuracy for both groups together was $92.3 \%$ (sensitivity (chronic low back pain classification) $=92.3 \%$; specificity (healthy controls) $=92.3 \%$ ). The clusters of brain activity that discriminated between the groups were located in the left somatosensory cortex (lower activity in chronic low back pain), and one in the left inferior parietal cortex (greater activity in chronic low back pain).

In another study, individuals with fibromyalgia were exposed to two different evoked task paradigms involving i) painful pressure, and ii) non-painful visual-auditory-tactile stimuli (Lopez-Sola et al., 2017). Correct patient versus control classification was achieved using either of these two paradigms or the neurological pain signature (i.e. pattern of brain activity associated with noxious thermal stimulation), with accuracies ranging between $68-89 \%$. However, combining these three signatures resulted in higher classification accuracy $(93 \%$; sensitivity $=92 \%$; specificity $=94 \%)$ in discriminating individuals with fibromyalgia from healthy controls.

A small study $(n=10)$ was unable to discriminate between individuals with temporomandibular pain and healthy controls when individuals were stimulated in the temporal or thumb region with a pressure stimulus (Harper et al., 2016). The average prediction accuracy was 60\% (Harper et al., 2016). However, in participants with temporomandibular disorder, MVPA was able to discriminate between evoked pain and rest (85.1\% prediction accuracy) (Harper et al., 2016). Pain processing regions such as the bilateral insula, anterior cingulate cortex and precuneus were regions of brain predictive of classifying pain versus rest (Harper et al., 2016) 
There is limited Level III-2 evidence that fMRI task inducing tasks, when combined with machinelearning can differentiate between chronic low back pain or fibromyalgia and healthy control participants (Grade of recommendation: C). There is limited Level III-2 evidence that fMRI with machine-learning cannot differentiate individuals with temporomandibular disorder from healthy control participants (Grade of recommendation: D).

\subsection{Predicting pain perception in healthy controls utilizing evoked pain paradigms with fMRI}

Various noxious stimuli were used to elicit experimental pain in 12 studies investigating areas of brain activity involved with the perception of pain.

\subsubsection{Laser Stimuli}

Two studies utilized laser stimuli to elicit pain (Brodersen et al., 2012; Liang, Mouraux, Hu, \& lannetti, 2013). In one study, laser stimuli (applied at an individual's pain threshold) was applied to the foot (Brodersen et al., 2012). It was demonstrated that whole-brain activity (determined by a linear trained and tested support vector machine) in the anticipation phase immediately prior to stimulus application (57.6\%) and during the stimulus period (61.4\%) was able to successfully predict the perception of pain. In the anticipation phase, the bilateral insula demonstrated the best predictive capacity, whilst brain activity in the mid cingulate cortex (MCC), somatosensory cortices (S1 \& S2), bilateral insula and orbitofrontal cortex successfully predicted the presence of pain perception during the stimulation period. Liang and colleagues (Liang et al., 2013) utilized fMRI with MVPA to investigate whether various sensory stimuli elicited spatial patterns of neuronal responses in the corresponding primary sensory cortex and in other primary sensory cortices. They demonstrated that laser stimuli applied to the dorsum of the foot was able to successfully predict the presence of pain in all primary sensory cortices, when compared to tactile, auditory or visual stimuli. In the primary somatosensory cortex (S1), MVPA classification demonstrated the ability to discriminate pain from the other sensory stimuli at accuracy rates ranging from 0.63-0.69. Similar results were obtained in the primary auditory cortex (0.59-0.83) and visual cortex (0.59-0.72).

In summary, two studies demonstrated that fMRI with MVPA could successfully predict the presence of pain following application of laser stimuli to the foot region in healthy controls with classification accuracy of approximately $60 \%$.

\subsubsection{Heat Stimuli}

Various studies $(n=8)$ utilized heat stimuli to elicit pain and train classifiers to investigate the ability of the classifiers to predict pain. Five studies utilized machine-learning techniques to predict the presence of acute pain within healthy individuals (Brodersen et al., 2012; Cecchi et al., 2012; Harper et al., 2016; Marquand et al., 2010; Ritter, Hebart, Wolbers, \& Bingel, 2014), whilst two studies used normative models developed in one cohort of participants to predict the presence of pain at an individual level in another cohort of participants (Brown, Chatterjee, Younger, \& Mackey, 2011; T. D. Wager et al., 2013). Two studies applied heat to the right palm or forearm region (CorradiDell'Acqua et al., 2011; Marquand et al., 2010), five studies applied it to the left forearm (Brown et al., 2011; L. J. Chang et al., 2015; Krishnan et al., 2016; Ritter et al., 2014; T. D. Wager et al., 2013), and one study applied this to the low back region (Cecchi et al., 2012).

The first study to use MVPA on whole-brain $\mathrm{FMRI}$ in the context of pain evaluated the predictive capability of different models in healthy individuals to predict self-reported thermal pain (Marquand et al., 2010). Heat stimuli were applied to the volar surface of the right forearm at different thresholds (sensory detection threshold; pain detection threshold and pain tolerance threshold). 
Support vector machines were able to accurately (72.3\%) discriminate between sensory and pain detection thresholds.

In another study, fMRI was used with multivariate pattern analysis to determine if regions of the brain associated with 'feeling' noxious stimuli were identical to those 'seeing' hand images in painful situations (Corradi-Dell'Acqua et al., 2011). The classifier (linear support vector machine) was trained to discriminate painful from non-painful heat stimulation and then tested to determine if it could distinguish between painful and non-painful observed hand images. The analysis revealed that there were shared patterns of brain activity in the right anterior insula for both felt pain and seen pain.

Another study used fMRI and MVPA to test whether spatial information of painful stimuli was represented in the descending pain modulatory system (periacqueductal gray, dorsolateral prefrontal cortex and rostral anterior cingulate cortex) (Ritter et al., 2014). Individual heat pain thresholds were determined for the left inner forearm and lateral calf. A linear support vector machine (LIBSVM (C. C. Chang \& Lin, 2011)) was trained and tested to determine how accurately it could distinguish between stimulation of the arm or leg in the respective brain region of interests. During both anticipation and painful stimulation, the site of nociceptive stimulation (arm or leg) could be successfully decoded from brain activity in the rostral anterior cingulate cortex (88-90\%), bilateral dorsolateral prefrontal cortex (79-83\%) and contralateral parietal operculum (84-91\%). The peracqueductal gray did not allow for classification above chance level.

Brown and colleagues (2011) were the first authors to train a linear support vector machine classifier on $\mathrm{fMRI}$ data to determine between-subject predictions (Brown et al., 2011). They applied a noxious heat stimulus to the left forearm on an initial eight individuals. This was then tested on 16 individuals and was $81 \%$ accurate in classifying painful from non-painful heat. Performance of the support vector machine was involved with brain activity in the somatosensory cortices (S1, S2), insular cortex, primary motor cortex (M1) and cingulate cortex. When compared to univariate analysis of single region of interest brain activity, S2 (71.9\%) and mid-insular cortex (64.3\%), MVPA performed at better than chance level in differentiating between painful or non-painful stimuli. Another study compared predictive models trained on both psychophysical and also brain activity to predict the presence of pain whilst delivering heat stimuli to the back region (Cecchi et al., 2012). They trained two predictive linear models to infer pain ratings from fMRI traces, but also utilized a combined model (trained on the same data) to also infer temperature from brain traces, combining both (pain and temperature) predictions into one model. The combined model demonstrated increased predictive accuracy for pain perception, close to $75 \%$ averaged over participants, when compared to the other models tested.

Wager et al performed four studies to develop an fMRI-based measure to predict pain intensity at an individual level (Tor D. Wager et al., 2013). Relevant brain areas were chosen from studies in the NeuroSynth meta-analysis database that used the word 'pain'. The neurological pain signature was derived as a result of a machine-learning algorithm that predicted pain reports from fMRI activity across brain regions whilst individuals underwent heat stimulation to the left forearm. When discriminating between painful heat and non-painful warmth, or pain anticipation or pain recall, the neurological pain signature demonstrated sensitivity and specificity of greater than $94 \%$. This signature was tested using another MRI scanner. In this study, the neurological pain signature was able to distinguish between painful heat and non-painful warmth with $93 \%$ sensitivity and specificity, whilst in a further study on another sample of healthy controls, the neurological pain signature demonstrated $85 \%$ sensitivity and $78 \%$ specificity. The neurological pain signature involved bilateral brain activation in the dorsal posterior insula, anterior insula, ventrolateral thalamus, medial thalamus, hypothalamus, dorsal anterior cingulate cortex and somatosensory cortex (S2), and 
contralateral somatosensory cortex ( $\mathrm{S} 1$ and $\mathrm{S} 2$ ) involvement. Using some of the same sample as the above study (40 of the 60 participants were common), the neurological pain signature demonstrated 92\% sensitivity in discriminating between heat-pain and warmth (Woo et al., 2014).

In summary, a series of studies performed by Wager and colleagues have showed that the neurological pain signature can demonstrate the ability to successfully discriminate painful heat from non-painful warmth in different scanners and different healthy populations with accuracy rates ranging between $70-94 \%$ (Tor D. Wager et al., 2013).

\subsubsection{Pressure Stimuli}

One small study $(n=10)$ utilized noxious pressure stimuli in the temporalis region (Harper et al., 2016). The authors demonstrated that MVPA predicted evoked pain versus rest within healthy individuals (83.3\% accuracy) (Harper et al., 2016).

\subsubsection{Visual Images}

Four studies investigated the ability of $\mathrm{fMRI}$ and machine classifiers to discriminate between 'somatic pain' (as elicited by a noxious thermal stimulus) and non-noxious 'social pain' (visualization of distressing images) (L. J. Chang et al., 2015; Krishnan et al., 2016; Tor D. Wager et al., 2013; Woo et al., 2014). Wager and colleagues investigated the specificity of the neurological pain signature by applying it to a group of individuals who recently underwent a break-up with a loved one ('rejected').

In this study, individuals were shown images of their ex-partner ('rejected') and also a close 'friend'. In discriminating between 'somatic pain' and 'social pain', the neurological pain signature demonstrated $85 \%$ sensitivity and $73 \%$ specificity. The neurological pain signature was unable to predict at better than chance level for 'rejector' and 'friend' conditions.

In another study utilizing some of the same cohort $(n=40)$ as the above study, the authors investigated whether representations of pain and rejection were common or distinct (Woo et al., 2014). In this sample $(n=60)$, the neurological pain signature was able to discriminate between heat-pain and ex-partner pictures with $100 \%$ accuracy. It performed at chance for ex-partner versus friend (accuracy $=59 \%$ ). Activation that was more strongly predictive of pain occurred in the supramarginal gyrus, middle insula, dorsolateral posterior insula and ventral insula, periacqueductal gray, amygdale and thalamus. Two other studies developed neural signatures, sensitive and specific for either picture-induced negative affect (PINES) (C. C. Chang \& Lin, 2011) or vicarious pain (Krishnan et al., 2016). These studies demonstrated that both of these signatures were not responsive to 'somatic pain' but specific to 'negative emotion/vicarious pain' (93\%/71\% discriminative accuracy), and conversely, the neurological pain signature was able to discriminate 'physical pain' from 'emotion/vicarious pain' (89.3\%/89\% discriminative accuracy), indicating that the respective signatures capture distinct aversive states.

There is Level III-2 evidence that machine-learning classifiers may be able to successfully discriminate between pain elicited by noxious heat stimuli and either warmth or non-noxious visual images (Grade of recommendation: $\mathrm{C}$ ).

\section{Discussion:}

This systematic review aimed to determine if MVPA, when used with structural or functional MRI, could discriminate between a) individuals with musculoskeletal pain and healthy controls, and $b$ ) healthy individuals stimulated with a noxious stimulus compared to a non-noxious control. These results complement and are in agreement with a recent narrative review on biomarkers in MSK pain 
conditions (Boissoneault, Sevel, Letzen, Robinson, \& Staud, 2017). Overall, the review demonstrated that there is preliminary evidence that MVPA can discriminate between individuals with musculoskeletal pain and healthy controls with an overall accuracy ranging from 53\% to $94 \%$. Specifically, structural brain abnormalities demonstrated classification accuracies ranging from $53.3 \%$ to $94.2 \%$; whilst fMRI task-evoked studies ranged from $60 \%$ to $93 \%$. Lastly, resting-state fMRI showed classification accuracies in the range of $57 \%$ to $62 \%$. The studies agree that MVPA provides insight into the central pathophysiological mechanisms evident at an individual level that are associated with pain, which have previously not been available to investigate. However, significant heterogeneity in patient conditions, study methodology and brain imaging techniques resulted in various findings that make study comparisons and formal conclusions challenging, resulting in the overall level of evidence being 'satisfactory' (Level III-2). There were other important findings evident that also warrant discussion.

\section{Structural MRI}

Studies investigating grey matter density demonstrated $>75 \%$ sensitivity and specificity in distinguishing chronic low back pain from healthy control participants (Baliki et al., 2011; Ung et al., 2014), whilst a study investigating changes in brain volumes demonstrated that accuracy for classifying individuals as fibromyalgia or healthy ranged from 53 to $76 \%$, but was not as accurate as self-report (Robinson et al., 2015). Comparison between studies was challenging as only two studies compared a similar patient condition (Baliki et al., 2011; Ung et al., 2014), whilst different methodologies were utilized to discriminate between groups. Unfortunately, only one study (Ung et al., 2014) reported the multivariate brain region findings which discriminated between low back pain and healthy controls; that being decreased grey matter density in right amygdala, left medial orbital gyrus and right cuneus, together with increased grey matter density in right dorsolateral prefrontal cortex, right cerebellum, left fusiform gyrus, multiple temporal lobe regions, left somatosensory cortices (S1/S2), left primary motor cortex (M1) and right primary visual cortex (V1). Traditionally, it has been reported that a decrease in grey matter density discriminates between patients and controls (Apkarian et al., 2004). However, it has been shown that individuals with persistent pain awaiting hip arthroplasty (Gwilym, Filippini, Douaud, Carr, \& Tracey, 2010) or with persistent headache following whiplash injury (Obermann et al., 2009) demonstrate increased grey matter density in different brain regions. Differences in grey matter density for chronic pain conditions are not uncommon (Cauda et al., 2014), with a recent systematic review of grey matter changes in chronic low back pain indicating that there was significant variation in the changes evident (Kregel et al., 2015). Different scanning parameters, learning algorithms and statistical thresholding could also have contributed to these findings. The results suggest that in longstanding low back pain, knee osteoarthritis and fibromyalgia that there is a convergence of evidence available to discriminate patients from healthy control participants using structural MRI.

\section{Functional MRI - Patient Populations}

Four studies investigated whether fMRI with machine-learning algorithms can discriminate between patient groups and healthy controls. One study investigated resting state functional connectivity, reporting classification accuracies of 57-62\% (Sundermann et al., 2014), whereas the other three studies utilized task based fMRI (with diagnostic accuracies ranging from 60-93\%) (Callan et al., 2014; Harper et al., 2016; Lopez-Sola et al., 2016). The study utilizing resting state functional connectivity was unable to significantly discriminate patients with fibromyalgia from healthy controls (Sundermann et al., 2014). The authors postulated that this may be due to low study numbers, and also as a result of the lack of functional connectivity alterations in the salience and default mode networks within fibromyalgia. The findings may also relate to the time of scan (5 minutes) performed. It has been demonstrated that longer duration scans (9 minutes) were required for the networks to be reliable (Van Dijk et al., 2010). Thus, fMRI studies applying MVPA to resting state 
connectivity data, with greater participant numbers are required to evaluate whether resting state functional organization is characteristically altered in chronic pain patients. Although the task based MRIs were successfully able to discriminate individuals with chronic low back pain (Callan et al., 2014) or fibromyalgia (Lopez-Sola et al., 2016) from healthy controls; apart from reduced response in the primary somatosensory cortex (which is associated with the sensory discriminative nature of pain); the brain regions responsible for discrimination of disease-state individuals differed between the studies. As univariate analyses also fail to demonstrate consistent differences between healthy and disease-state individuals (Baliki et al., 2006; Giesecke et al., 2004), it suggests that there are overlapping patterns of brain function for different chronic pain conditions (Apkarian et al., 2011). Methodological differences related to evoked task/stimuli utilized; MRI scanner, acquisition and preprocessing, and feature reduction and classifier selection may also be responsible for the betweenstudy differences in patterns of regional brain activation findings. At the very minimum, consistent feature reduction and classifier selection would enhance comparison between study findings. Thus, emerging evidence from a limited number of studies suggests that MVPA of task-based fMRI may be able to differentiate individuals with patient conditions from healthy control participants, whereas functional connectivity analyses of the salience network and default mode network cannot currently differentiate fibromyalgia patients from healthy controls.

\section{Functional MRI - Healthy Individuals}

The majority of studies $(n=12)$ reviewed focussed on the ability of $f M R I$, when paired with machine learning algorithms to discriminate between healthy individuals stimulated with a noxious stimulus, and those presented with a non-noxious control. Statistical pooling was not possible due to significant heterogeneity resulting from various fMRI design study methodologies employed (Supplementary Table 2) and variability in participant demographics. Despite these limitations, the studies were consistent in reporting that MVPA was successfully able to discriminate between a noxious and non-noxious stimulus that was either not evident via univariate analyses (Ritter et al., 2014), or with a greater degree of accuracy than that provided by univariate analyses (Brodersen et al., 2012; Brown et al., 2011), with 'pain' detection rates ranging from $60 \%$ to $94 \%$ for thermal- or laser-evoked noxious stimulation. Given that single brain regions traditionally associated with pain are also associated with other functions such as touch, attention, salience, emotion, and other cognitive and sensorimotor functions (lannetti, Salomons, Moayedi, Mouraux, \& Davis, 2013), MVPA has illustrated the benefit of evaluating 'patterns' of brain function when investigating pain. Unfortunately, apart from one study utilizing noxious pressure that demonstrated similarly high discriminatory values (84\%) (Harper et al., 2016), limited studies have evaluated brain activity resulting from other possible pain induction techniques, of which there are many (Curatolo, Petersen-Felix, \& Arendt-Nielsen, 2004). As no studies have compared brain activity for different pain induction modalities, it is currently unclear if different modalities result in similar or different patterns of brain activity.

Although the studies in this review demonstrated general agreement that brain regions which discriminate between noxious and non-noxious stimulation belong to classical pain processing regions (Brodersen et al., 2011; Brown et al., 2011), these results also demonstrate that other brain regions, such as the primary visual cortex may also assist in delineating the presence of a noxious stimulus (Liang et al., 2013; T. D. Wager et al., 2013), supporting the idea that pain does not reside in any one particular part of the brain (Apkarian, Bushnell, Treede, \& Zubieta, 2005). Thus, these findings expand on the traditional 'pain matrix' previously observed in structural and fMRI studies involving univariate analyses (Apkarian et al., 2005) and are in line with the recent proposal of a dynamic pain connectome (Kucyi \& Davis, 2015), suggesting that pain is not only associated with ascending nociceptive pathways (such as somatosensory, insular, cingulate and prefrontal cortices), but is also associated with brain networks involving attention and salience and the default mode network. These MVPA findings also, not only illustrate the presence of a distributed pain network 
upon noxious stimulation, but also highlight the activation of different populations of neurons in differing pain percepts (Liang et al., 2013; Woo et al., 2014) and emotional states (Krishnan et al., 2016; T. D. Wager et al., 2013; Woo et al., 2014).

\section{Other Findings/Implications:}

The utility of MVPA is not only demonstrated in delineation between noxious and non-noxious stimuli. MVPA has also demonstrated an ability to understand the spatial representation of pain processing. It has been shown that the site of noxious stimulus (arm or leg; temporalis or thumb) can be decoded, within brain regions investigated in both healthy individuals (Ritter et al., 2014), and also in patients (Harper et al., 2016). MVPA has also been used to demonstrate the similarities and differences in brain findings between the percept of pain ('seen') and the feeling of pain (Corradi-Dell'Acqua et al., 2011; Krishnan et al., 2016). This review also revealed that improvements in patient group classification accuracy and pain prediction are possible, either with model optimization (Sundermann et al., 2014), or utilization of combined models of prediction, such as utilizing behavioural measures (rather than relying on features of neuroimaging alone) (Cecchi et al., 2012). Combining neurological signatures that were associated with different pain symptoms present in an individual's pain experience, also increased diagnostic accuracy for discriminating between healthy and disease states (Lopez-Sola et al., 2016). Thus, this review highlighted that different structural and fMRI paradigms possibly capture different processes that underlie an individual's MSK pain experience. And so, different structural and fMRI paradigms, if designed and tuned appropriately, may be helpful in disentangling different component processes in each MSK pain condition and even across MSK pain conditions. This may help to further identify component processes that are common to various MSK pain conditions and component processes which are more specific to one or other MSK pain conditions. It would make sense that studying the brain in a tailored and comprehensive manner, as reviewed here, will assist in disentangling the commonalities and specificities across MSK pain conditions and may be helpful in allowing detection of objective patient subtypes based on measurable alterations in specific and previously characterized brain processes (Lopez-Sola et al., 2016).

\section{Limitations and Future Directions}

Although these emerging findings are promising, significant limitations remain. It is evident that there is currently no current single imaging-based neural signature for chronic pain. As chronic pain is present without need for an evoked stimulus, there may be a requirement for diagnostic imaging procedures that do not involve task-evoked fMRI paradigms. Procedures such as resting state functional connectivity or perfusion methods such as arterial spin labelling or positron emission tomography might assist in this regard. Thus, utilization of different, and possibly combined neuroimaging paradigms may assist in providing evaluation of pathophysiologically significant processes, which should provide a more complete characterization of patient status, than what may be provided with one paradigm alone. The findings in this review were also limited to small studies with younger and predominantly female participants, and have largely not been validated in clinical or chronic pain conditions where various emotional states commonly associated with pain co-exist (e.g. distress, anxiety, stress, depression). The presence of these co-morbidities in various MSK pain states will likely affect the ability of MVPA to distinguish between patients, although Lopez-Sola and colleagues utilized a combination of neurological signatures to accurately discriminate between fibromyalgia and healthy controls (Lopez-Sola et al., 2016). No prospective studies were found in this review to allow determination of the prognostic or diagnostic capabilities or treatment responsiveness of these analyses. Future studies would also benefit from combining various behavioural, genotype and phenotype data into analyses to assist with development of sensitive and specific signatures that could guide future individualized patient treatment options and evaluate how treatments exert their effects. 
In conclusion, there is preliminary and emerging evidence that MVPA analyses of structural or functional MRI are able to discriminate between patients and healthy controls, and also discriminate between noxious and non-noxious stimulation. However, further studies are required to overcome current limitations in different patient populations to assist with further translation of these results to determine possible clinical utility. 


\section{References:}

Apkarian, A. V. (2011). The brain in chronic pain: clinical implications. Pain Manag, 1(6), 577-586. doi:10.2217/pmt.11.53

Apkarian, A. V., Bushnell, M. C., Treede, R. D., \& Zubieta, J. K. (2005). Human brain mechanisms of pain perception and regulation in health and disease. Eur J Pain, 9(4), 463-484. doi:10.1016/j.ejpain.2004.11.001

Apkarian, A. V., Hashmi, J. A., \& Baliki, M. N. (2011). Pain and the brain: specificity and plasticity of the brain in clinical chronic pain. Pain, 152(3 Suppl), S49-64. Retrieved from http://www.ncbi.nlm.nih.gov/entrez/query.fcgi?cmd=Retrieve\&db=PubMed\&dopt=Citation \&list uids $=21146929$

http://graphics.tx.ovid.com/ovftpdfs/FPDDNCOBMFEPIN00/fs047/ovft/live/gv024/00006396/00006 396-201103001-00008.pdf

Apkarian, A. V., Sosa, Y., Sonty, S., Levy, R. M., Harden, R. N., Parrish, T. B., \& Gitelman, D. R. (2004). Chronic back pain is associated with decreased prefrontal and thalamic gray matter density. J Neurosci, 24(46), 10410-10415. doi:10.1523/JNEUROSCI.2541-04.2004

Baliki, M. N., Chialvo, D. R., Geha, P. Y., Levy, R. M., Harden, R. N., Parrish, T. B., \& Apkarian, A. V. (2006). Chronic pain and the emotional brain: specific brain activity associated with spontaneous fluctuations of intensity of chronic back pain. J Neurosci, 26(47), 12165-12173. doi:10.1523/jneurosci.3576-06.2006

Baliki, M. N., Petre, B., Torbey, S., Herrmann, K. M., Huang, L., Schnitzer, T. J., . . . Apkarian, A. V. (2012). Corticostriatal functional connectivity predicts transition to chronic back pain. Nat Neurosci, 15(8), 1117-1119. Retrieved from http://www.ncbi.nlm.nih.gov/entrez/query.fcgi?cmd=Retrieve\&db=PubMed\&dopt=Citation \&list uids $=22751038$

http://www.ncbi.nlm.nih.gov/pmc/articles/PMC3411898/pdf/nihms383388.pdf

Baliki, M. N., Schnitzer, T. J., Bauer, W. R., \& Apkarian, A. V. (2011). Brain morphological signatures for chronic pain. PLoS One, 6(10), e26010. doi:10.1371/journal.pone.0026010

Baron, R., Binder, A., \& Wasner, G. (2012). Neuropathic pain: diagnosis, pathophysiological mechanisms, and treatment. Lancet Neurol, 9(8), 807-819. Retrieved from http://www.ncbi.nlm.nih.gov/entrez/query.fcgi?cmd=Retrieve\&db=PubMed\&dopt=Citation $\underline{\text { \&list uids }=20650402}$

http://media.proquest.com/media/pq/classic/doc/2088566731/fmt/pi/rep/NONE?hl=\&cit\%3Aauth= Baron\%2C+Ralf\%3BBinder\%2C+Andreas\%3BWasner\%2C+Gunnar\&cit\%3Atitle=Neuropathict pain\%3A+diagnosis\%2C+pathophysiological+mechanisms\%2C+and+treatment\&cit\%3Apub=T he+Lancet+Neurology\&cit\%3Avol=9\&cit\%3Aiss=8\&cit\%3Apg=807\&cit\%3Adate=Aug+2010\&i c=true\&cit\%3Aprod=ProQuest+Central\& a=ChgyMDE1MDUyMDIyNTcwNTI3Nzo5NDI5ODAS BTk2MDA1GgpPTkVfUOVBUkNIIg0xMzluMjMOLjlyOC43KgUyNjl1NTIJNjM1OTc2NzE4Og1Eb2 N1bWVudEItYWdlQgEwUgZPbmxpbmVaAkZUYgNQRIRqCilwMTAvMDgvMDFyCilwMTAvMDg vMzF6AIIBMIAtMTAwNzA2Ny0xNDU0My1DVVNUT01FUiOxMDAwMDAzOS8xMDAwMDE1NS OxMjUxNjYxkgEGT25saW5lygEHRW5kTm90ZdIBEINjaG9sYXJseSBKb3VybmFsc5oCB1ByZVBha WSqAihPUzpFTVMtUGRmRG9jVmlldOJhc2UtZ2V0TWVkaWFVcmxGb3JJdGVtygIPRmVhdHVyZ XxBcnRpY2x|0g|BWelCAU7yAgA\%3D\& s=Gldb\%2B9e5bVKreK4d2yDFeWH56Fc\%3D

Boissoneault, J., Sevel, L., Letzen, J., Robinson, M., \& Staud, R. (2017). Biomarkers for Musculoskeletal Pain Conditions: Use of Brain Imaging and Machine Learning. Curr Rheumatol Rep, 19(1), 5. doi:10.1007/s11926-017-0629-9

Brodersen, K. H., Haiss, F., Ong, C. S., Jung, F., Tittgemeyer, M., Buhmann, J. M., . . . Stephan, K. E. (2011). Model-based feature construction for multivariate decoding. Neuroimage, 56(2), 601-615. doi:10.1016/j.neuroimage.2010.04.036 
Brodersen, K. H., Wiech, K., Lomakina, E. I., Lin, C. S., Buhmann, J. M., Bingel, U., ... Tracey, I. (2012). Decoding the perception of pain from $\mathrm{fMRI}$ using multivariate pattern analysis. Neuroimage, 63(3), 1162-1170. doi:10.1016/j.neuroimage.2012.08.035

Brown, J. E., Chatterjee, N., Younger, J., \& Mackey, S. (2011). Towards a physiology-based measure of pain: patterns of human brain activity distinguish painful from non-painful thermal stimulation. Plos One, 6(9), e24124. doi:10.1371/journal.pone.0024124

Callan, D., Mills, L., Nott, C., England, R., \& England, S. (2014). A tool for classifying individuals with chronic back pain: using multivariate pattern analysis with functional magnetic resonance imaging data. PLoS One, 9(6), e98007. doi:10.1371/journal.pone.0098007

Cauda, F., Palermo, S., Costa, T., Torta, R., Duca, S., Vercelli, U., . . Torta, D. M. (2014). Gray matter alterations in chronic pain: A network-oriented meta-analytic approach. Neuroimage Clin, 4, 676-686. doi:10.1016/j.nicl.2014.04.007

Cecchi, G. A., Huang, L., Hashmi, J. A., Baliki, M., Centeno, M. V., Rish, I., \& Apkarian, A. V. (2012). Predictive dynamics of human pain perception. Plos Computational Biology, 8(10), e1002719-e1002719. doi:10.1371/journal.pcbi.1002719

Chang, C. C., \& Lin, C. J. (2011). LIBSVM: A Library for Support Vector Machines. ACM Transactions on Intelligent Systems and Technology, 2(3), 21-27. doi:Artn 27

10.1145/1961189.1961199

Chang, L. J., Gianaros, P. J., Manuck, S. B., Krishnan, A., \& Wager, T. D. (2015). A Sensitive and Specific Neural Signature for Picture-Induced Negative Affect. PLoS Biol, 13(6), e1002180. doi:10.1371/journal.pbio.1002180

Clark, W. C., Yang, J. C., Tsui, S. L., Ng, K. F., \& Bennett Clark, S. (2002). Unidimensional pain rating scales: a multidimensional affect and pain survey (MAPS) analysis of what they really measure. Pain, 98(3), 241-247. Retrieved from http://www.ncbi.nlm.nih.gov/pubmed/12127025

Corradi-Dell'Acqua, C., Hofstetter, C., \& Vuilleumier, P. (2011). Felt and seen pain evoke the same local patterns of cortical activity in insular and cingulate cortex. The Journal Of Neuroscience: The Official Journal Of The Society For Neuroscience, 31(49), 17996-18006. doi:10.1523/JNEUROSCI.2686-11.2011

Curatolo, M., Petersen-Felix, S., \& Arendt-Nielsen, L. (2004). Assessment of regional analgesia in clinical practice and research. Br Med Bull, 71, 61-76. Retrieved from http://www.ncbi.nlm.nih.gov/entrez/query.fcgi?cmd=Retrieve\&db=PubMed\&dopt=Citation $\underline{\text { \&list uids }=15684246}$

http://bmb.oxfordjournals.org/content/71/1/61.full.pdf

Davis, T., LaRocque, K. F., Mumford, J. A., Norman, K. A., Wagner, A. D., \& Poldrack, R. A. (2014). What do differences between multi-voxel and univariate analysis mean? How subject-, voxel-, and trial-level variance impact fMRI analysis. Neuroimage, 97, 271-283. doi:10.1016/j.neuroimage.2014.04.037

Editors, T. P. M. (2014). Observational studies: getting clear about transparency. PLoS Med, 11(8), e1001711. doi:10.1371/journal.pmed.1001711

Flor, H., Braun, C., Elbert, T., \& Birbaumer, N. (1997). Extensive reorganization of primary somatosensory cortex in chronic back pain patients. Neurosci Lett, 224(1), 5-8. Retrieved from http://www.ncbi.nlm.nih.gov/entrez/query.fcgi?cmd=Retrieve\&db=PubMed\&dopt=Citation \&list uids $=9132689$

http://ac.els-cdn.com/S0304394097134413/1-s2.0-S0304394097134413-main.pdf? tid=acd0e928ff49-11e4-938a-00000aab0f02\&acdnat=1432165442 296f1cf0dd98defa23f7d98a078b423e 
Giesecke, T., Gracely, R. H., Grant, M. A., Nachemson, A., Petzke, F., Williams, D. A., \& Clauw, D. J. (2004). Evidence of augmented central pain processing in idiopathic chronic low back pain. Arthritis Rheum, 50(2), 613-623. doi:10.1002/art.20063

Gwilym, S. E., Filippini, N., Douaud, G., Carr, A. J., \& Tracey, I. (2010). Thalamic atrophy associated with painful osteoarthritis of the hip is reversible after arthroplasty: a longitudinal voxelbased morphometric study. Arthritis Rheum, 62(10), 2930-2940. doi:10.1002/art.27585

Harper, D. E., Shah, Y., Icheso, E., Gerstner, G., \& Peltier, S. (2016). Multivariate classification of painevoked brain activity in temporomandibular disorder. Pain Reports, 1(3), e572. doi:10.1097/PR9.0000000000000572

Haufe, S., Meinecke, F., Gorgen, K., Dahne, S., Haynes, J. D., Blankertz, B., \& Biessmann, F. (2014). On the interpretation of weight vectors of linear models in multivariate neuroimaging. Neuroimage, 87, 96-110. doi:10.1016/j.neuroimage.2013.10.067

Haxby, J. V., Gobbini, M. I., Furey, M. L., Ishai, A., Schouten, J. L., \& Pietrini, P. (2001). Distributed and overlapping representations of faces and objects in ventral temporal cortex. Science, 293(5539), 2425-2430. doi:10.1126/science.1063736

Haynes, J. D., \& Rees, G. (2006). Decoding mental states from brain activity in humans. Nat Rev Neurosci, 7(7), 523-534. doi:10.1038/nrn1931

lannetti, G. D., Salomons, T. V., Moayedi, M., Mouraux, A., \& Davis, K. D. (2013). Beyond metaphor: contrasting mechanisms of social and physical pain. Trends Cogn Sci, 17(8), 371-378. doi:10.1016/j.tics.2013.06.002

Kregel, J., Meeus, M., Malfliet, A., Dolphens, M., Danneels, L., Nijs, J., \& Cagnie, B. (2015). Structural and functional brain abnormalities in chronic low back pain: A systematic review( 3 ). Semin Arthritis Rheum. doi:10.1016/j.semarthrit.2015.05.002

Krishnan, A., Woo, C. W., Chang, L. J., Ruzic, L., Gu, X., Lopez-Sola, M., ... Wager, T. D. (2016). Somatic and vicarious pain are represented by dissociable multivariate brain patterns. Elife, 5. doi:10.7554/eLife.15166

Kucyi, A., \& Davis, K. D. (2015). The dynamic pain connectome. Trends Neurosci, 38(2), 86-95. doi:10.1016/j.tins.2014.11.006

Liang, M., Mouraux, A., Hu, L., \& lannetti, G. D. (2013). Primary sensory cortices contain distinguishable spatial patterns of activity for each sense. Nat Commun, 4, 1979. doi:10.1038/ncomms2979

Lopez-Sola, M., Woo, C. W., Pujol, J., Deus, J., Harrison, B. J., Monfort, J., \& Wager, T. D. (2016). Towards a neurophysiological signature for fibromyalgia. Pain. doi:10.1097/j.pain.0000000000000707

Lopez-Sola, M., Woo, C. W., Pujol, J., Deus, J., Harrison, B. J., Monfort, J., \& Wager, T. D. (2017). Towards a neurophysiological signature for fibromyalgia. Pain, 158(1), 34-47. doi:10.1097/j.pain.0000000000000707

Mackey, S. C. (2013). Central neuroimaging of pain. J Pain, 14(4), 328-331. doi:10.1016/j.jpain.2013.01.001

Marquand, A., Howard, M., Brammer, M., Chu, C., Coen, S., \& Mourão-Miranda, J. (2010). Quantitative prediction of subjective pain intensity from whole-brain fMRI data using Gaussian processes. Neuroimage, 49(3), 2178-2189. doi:10.1016/j.neuroimage.2009.10.072

Mur, M., Bandettini, P. A., \& Kriegeskorte, N. (2009). Revealing representational content with pattern-information fMRI--an introductory guide. Soc Cogn Affect Neurosci, 4(1), 101-109. doi:10.1093/scan/nsn044

Obermann, M., Nebel, K., Schumann, C., Holle, D., Gizewski, E. R., Maschke, M., . . Katsarava, Z. (2009). Gray matter changes related to chronic posttraumatic headache. Neurology, 73(12), 978-983. doi:10.1212/WNL.0b013e3181b8791a

Poldrack, R. A., Fletcher, P. C., Henson, R. N., Worsley, K. J., Brett, M., \& Nichols, T. E. (2008). Guidelines for reporting an fMRI study. Neuroimage, 40(2), 409-414. doi:10.1016/j.neuroimage.2007.11.048 
Ritter, C., Hebart, M. N., Wolbers, T., \& Bingel, U. (2014). Representation of spatial information in key areas of the descending pain modulatory system. The Journal Of Neuroscience: The Official Journal Of The Society For Neuroscience, 34(13), 4634-4639. doi:10.1523/JNEUROSCI.4342-13.2014

Robinson, M. E., O'Shea, A. M., Craggs, J. G., Price, D. D., Letzen, J. E., \& Staud, R. (2015). Comparison of machine classification algorithms for fibromyalgia: neuroimages versus self-report. J Pain, 16(5), 472-477. doi:10.1016/j.jpain.2015.02.002

Rogachov, A., Cheng, J. C., \& DeSouza, D. D. (2015). Discriminating neural representations of physical and social pains: how multivariate statistics challenge the "shared representation" theory of pain. J Neurophysiol, 114(5), 2558-2560. doi:10.1152/jn.00075.2015

Schmidt-Wilcke, T., Leinisch, E., Ganssbauer, S., Draganski, B., Bogdahn, U., Altmeppen, J., \& May, A. (2006). Affective components and intensity of pain correlate with structural differences in gray matter in chronic back pain patients. Pain, 125(1-2), 89-97. doi:10.1016/j.pain.2006.05.004

Smallwood, R. F., Laird, A. R., Ramage, A. E., Parkinson, A. L., Lewis, J., Clauw, D. J., . . Robin, D. A. (2013). Structural brain anomalies and chronic pain: a quantitative meta-analysis of gray matter volume. J Pain, 14(7), 663-675. doi:10.1016/j.jpain.2013.03.001

Sundermann, B., Burgmer, M., Pogatzki-Zahn, E., Gaubitz, M., Stüber, C., Wessolleck, E., . . . Pfleiderer, B. (2014). Diagnostic classification based on functional connectivity in chronic pain: model optimization in fibromyalgia and rheumatoid arthritis. Academic Radiology, 21(3), 369-377. doi:10.1016/j.acra.2013.12.003

Tracey, I. (2011). Can neuroimaging studies identify pain endophenotypes in humans? Nat Rev Neurol, 7(3), 173-181. doi:10.1038/nrneurol.2011.4

Ung, H., Brown, J. E., Johnson, K. A., Younger, J., Hush, J., \& Mackey, S. (2014). Multivariate classification of structural MRI data detects chronic low back pain. Cerebral Cortex (New York, N.Y.: 1991), 24(4), 1037-1044. doi:10.1093/cercor/bhs378

Van Dijk, K. R., Hedden, T., Venkataraman, A., Evans, K. C., Lazar, S. W., \& Buckner, R. L. (2010). Intrinsic functional connectivity as a tool for human connectomics: theory, properties, and optimization. J Neurophysiol, 103(1), 297-321. doi:10.1152/jn.00783.2009

Vos, T., Flaxman, A. D., Naghavi, M., Lozano, R., Michaud, C., Ezzati, M., . . Memish, Z. A. (2012). Years lived with disability (YLDs) for 1160 sequelae of 289 diseases and injuries 1990-2010: a systematic analysis for the Global Burden of Disease Study 2010. Lancet, 380(9859), 21632196. doi:10.1016/S0140-6736(12)61729-2

Wager, T. D., Atlas, L. Y., Lindquist, M. A., Roy, M., Woo, C.-W., \& Kross, E. (2013). An fMRI-based neurologic signature of physical pain. The New England Journal Of Medicine, 368(15), 13881397. doi:10.1056/NEJMoa1204471

Wager, T. D., Atlas, L. Y., Lindquist, M. A., Roy, M., Woo, C. W., \& Kross, E. (2013). An fMRI-based neurologic signature of physical pain. N Engl J Med, 368(15), 1388-1397. doi:10.1056/NEJMoa1204471

Woo, C. W., Koban, L., Kross, E., Lindquist, M. A., Banich, M. T., Ruzic, L., ... Wager, T. D. (2014). Separate neural representations for physical pain and social rejection. Nat Commun, 5, 5380. doi:10.1038/ncomms6380

Woolf, C. J. (2011). Central sensitization: implications for the diagnosis and treatment of pain. Pain, 152(3 Suppl), S2-15. Retrieved from http://www.ncbi.nlm.nih.gov/entrez/query.fcgi?cmd=Retrieve\&db=PubMed\&dopt=Citation \&list uids $=20961685$ 


\begin{tabular}{|c|c|c|c|c|c|c|c|c|c|c|c|c|c|c|c|}
\hline \multirow{2}{*}{$\begin{array}{l}\text { Study } \\
\text { Lead Author }\end{array}$} & \multicolumn{3}{|c|}{ Experimental Design } & \multicolumn{3}{|c|}{ Human Subjects } & \multirow{2}{*}{$\begin{array}{l}\begin{array}{l}\text { Data } \\
\text { Acquisition }\end{array} \\
\text { Image } \\
\text { Properties - } \\
\text { as acquired }\end{array}$} & \multicolumn{3}{|c|}{ Data Preprocessing } & \multicolumn{3}{|c|}{ Statistical Modeling } & \multicolumn{2}{|c|}{ Statistical Inference } \\
\hline & $\begin{array}{l}\text { Design } \\
\text { Specification }\end{array}$ & $\begin{array}{l}\text { Task } \\
\text { Specification }\end{array}$ & $\begin{array}{l}\text { Planned } \\
\text { Comparisons }\end{array}$ & $\begin{array}{l}\text { Details on } \\
\text { Subject } \\
\text { Sample }\end{array}$ & $\begin{array}{l}\text { Ethics } \\
\text { Approval }\end{array}$ & $\begin{array}{l}\text { Behavioural } \\
\text { Performance }\end{array}$ & & $\begin{array}{l}\text { Pre- } \\
\text { processing: } \\
\text { general }\end{array}$ & $\begin{array}{l}\text { Intersubject } \\
\text { registration }\end{array}$ & Smoothing & $\begin{array}{l}\text { General } \\
\text { Issues }\end{array}$ & $\begin{array}{l}\text { Intrasubject } \\
\text { fMRI } \\
\text { modelling } \\
\text { info }\end{array}$ & $\begin{array}{l}\text { Group } \\
\text { Modeling info }\end{array}$ & $\begin{array}{l}\text { Inference on } \\
\text { statistic image } \\
\text { (thresholding) }\end{array}$ & ROI analysis \\
\hline \multicolumn{16}{|c|}{ Patient Populations: } \\
\hline Callan & & $\mathrm{x}$ & $\mathrm{x}$ & $x$ & $\mathrm{x}$ & $\mathrm{X}$ & $x$ & $x$ & $x$ & $x$ & $x$ & $x$ & $x$ & $x$ & $\mathrm{~N} / \mathrm{A}$ \\
\hline Harper & & $x$ & $x$ & $x$ & $x$ & $x$ & $x$ & $x$ & $x$ & $x$ & & & & & N/A \\
\hline Lopez-Sola & & $x$ & $x$ & $x$ & $x$ & $\mathrm{X}$ & $x$ & $x$ & $\mathrm{x}$ & $x$ & $\mathrm{x}$ & $\mathrm{X}$ & $x$ & $x$ & $x$ \\
\hline Sundermann & & $\mathrm{N} / \mathrm{A}$ & $x$ & $x$ & $x$ & $\mathrm{X}$ & $x$ & $x$ & $x$ & $x$ & $\mathrm{x}$ & $x$ & $x$ & $x$ & $\mathrm{~N} / \mathrm{A}$ \\
\hline \multicolumn{16}{|c|}{ Healthy Participants: } \\
\hline Brodersen & $x$ & $\mathrm{x}$ & $x$ & $x$ & $\mathrm{x}$ & & $x$ & $x$ & $x$ & $x$ & $x$ & $x$ & $x$ & $x$ & $x$ \\
\hline Brown & & $\mathrm{x}$ & & $x$ & $x$ & & $x$ & $x$ & $x$ & $x$ & $\mathrm{x}$ & $x$ & $x$ & & $x$ \\
\hline Cecchi & & $x$ & & $x$ & $x$ & & $x$ & $x$ & $\mathrm{x}$ & $x$ & $\mathrm{x}$ & $\mathrm{x}$ & $x$ & & $\mathrm{~N} / \mathrm{A}$ \\
\hline Chang & $x$ & $x$ & $x$ & $x$ & $x$ & $x$ & $x$ & $x$ & $x$ & $x$ & $x$ & $x$ & $x$ & $x$ & $x$ \\
\hline $\begin{array}{l}\text { Corradi- } \\
\text { Dell-Acqua }\end{array}$ & $x$ & $x$ & $x$ & $x$ & $x$ & $x$ & $x$ & $x$ & $x$ & $x$ & $x$ & $x$ & $x$ & $x$ & $\mathrm{~N} / \mathrm{A}$ \\
\hline Krishnan & $x$ & $x$ & $x$ & $x$ & $x$ & & $x$ & $x$ & $x$ & $x$ & $x$ & $x$ & $x$ & $x$ & $x$ \\
\hline Liang & $x$ & $x$ & & $x$ & $x$ & & $x$ & $x$ & $x$ & & $x$ & $x$ & $x$ & $x$ & $x$ \\
\hline Marquand & $x$ & $x$ & & $x$ & $x$ & $x$ & $x$ & $x$ & $x$ & $x$ & $x$ & $x$ & $x$ & $x$ & $x$ \\
\hline Ritter & $x$ & $x$ & & $x$ & $x$ & $x$ & $x$ & $x$ & $x$ & $x$ & $x$ & & $x$ & & $x$ \\
\hline Wager & $x$ & $x$ & $x$ & $x$ & $x$ & & $x$ & $x$ & $x$ & $x$ & $x$ & $x$ & $x$ & $x$ & $\mathrm{x}$ \\
\hline Woo & $x$ & $x$ & $x$ & $x$ & $x$ & & $x$ & $x$ & $x$ & $x$ & $x$ & $x$ & $x$ & $x$ & N/A \\
\hline
\end{tabular}

\section{Supplementary Table 1 - Risk of Bias With fMRI Studies (Poldrack criteria)}




\section{Preprocessing}

Outlier/Gradient

Artifact

Slice

Acquisitio

Timing Image change normative

Correction Realignment conversion atlas

Software
Analysis:

Regressors

Constructed

\section{Prediction and Evaluation:}

Functional: Patient Populations

Callan

Harper

Lopez-Sola

Sundermann

\section{Functional: Healthy Participants}

Brodersen

Brown

Cecchi

Chang

Corradi-Dell-Acqua

Krishnan

Liang

Marquand

Ritter

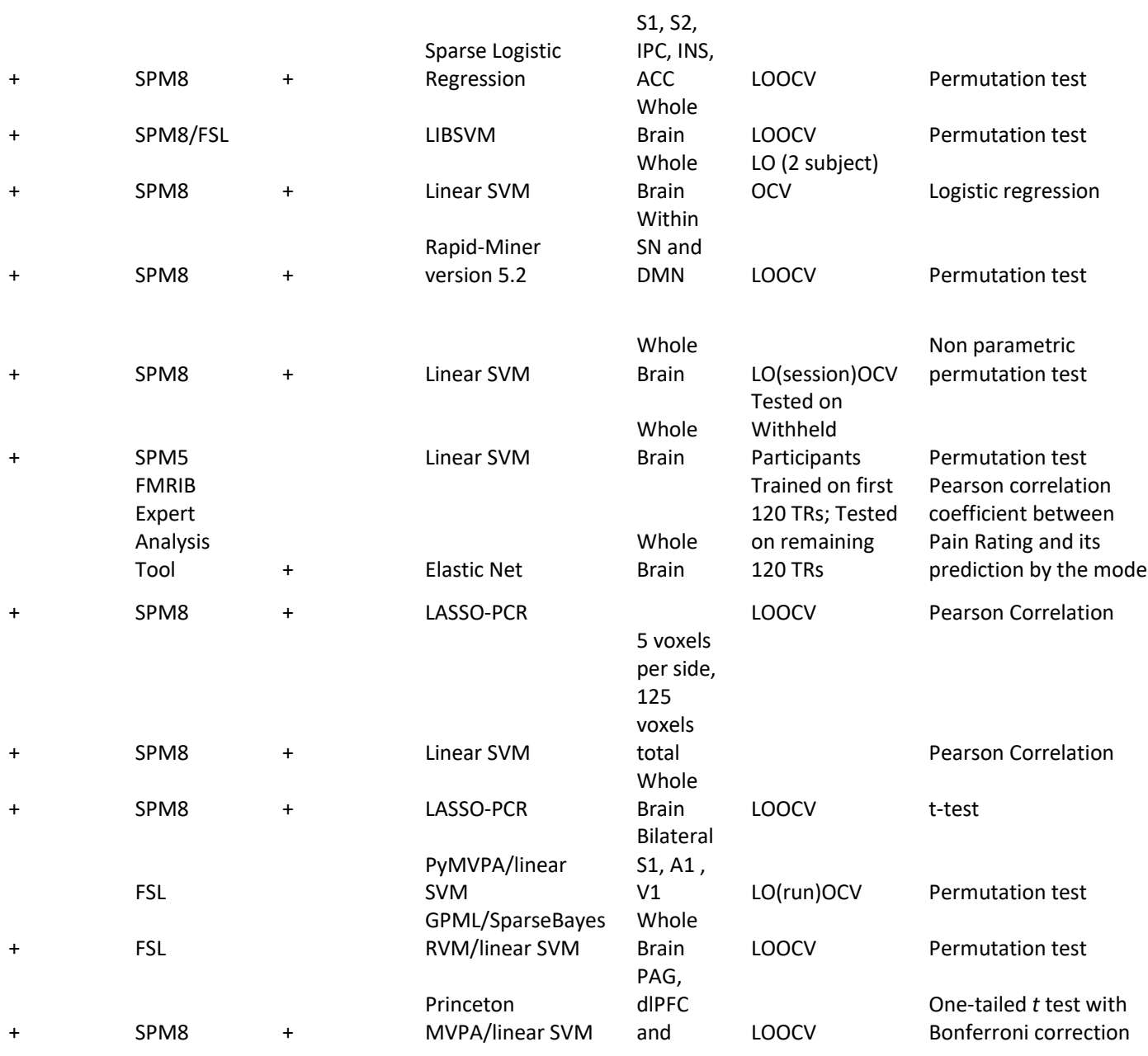




\begin{tabular}{|c|c|c|c|c|c|c|c|c|c|c|c|}
\hline Wager & + & + & + & + & + & SPM5/SPM8 & + & LASSO-PCR/FSL & $\begin{array}{l}\text { Whole } \\
\text { Brain }\end{array}$ & Bootstrapping & Permutation test \\
\hline Woo & + & + & + & + & + & SPM8 & + & Linear SVM & $\begin{array}{l}\text { Whole } \\
\text { Brain }\end{array}$ & LOOCV & $\begin{array}{l}\text { Multi-voxel Pattern } \\
\text { Similarity Analysis and } \\
\text { Cross Classification Test }\end{array}$ \\
\hline
\end{tabular}

Legend: SPM = Statistical Parametric Mapping; LOOCV = Leave-one-out Cross-Validation; S1 = primary somatosensory cortex; S2 = secondary somatosensory cortex; IPC = inferior parietal cortex; INS = insula; $\mathrm{ACC}=$ anterior cingulate cortex; $\mathrm{SN}$ = salience network; $\mathrm{DMN}=$ default mode network; LASSO-PCR = Least Absolute Shrinkage and Selection Operator-Principal Components Regression; $\mathrm{S1}=$ primary somatosensory cortex; $\mathrm{A} 1$ = primary auditory cortex; $\mathrm{V} 1$ = primary visual corex; GPML = Gaussian processes for machine learninng; PAG = Periaquductal gray; dIPFC = dorsolateral prefrontal cortex; rACC = rostral anterior cingulate cortex

\section{Supplementary Table 2 - fMRI Methodology Summaries}

